Pacific

Journal of

Mathematics

\title{
WOLFF SNOWFLAKES
}

John L. Lewis, Gregory C. Verchota And ANDrew L. Vogel 


\title{
WOLFF SNOWFLAKES
}

\author{
John L. LEWIS, GRegory C. Verchota AND ANDREW L. Vogel
}

Dedicated to the memory of Thomas $H$. Wolff, a true mathematical giant.

\section{We study the dimension of harmonic measure for Wolff snowflakes.}

\section{Introduction}

Denote points in Euclidean $n$-space $\mathbb{R}^{n}$ by $X=(x, t)$, with $x=\left(x_{1}, \ldots, x_{n-1}\right) \in$ $\mathbb{R}^{n-1}$ and $t \in \mathbb{R}$, and let $d x$ and $d X=d x d t$ denote Lebesgue measure on $\mathbb{R}^{n-1}$ and $\mathbb{R}^{n}$. Let $|X|$ be the usual Euclidean norm of $X$ and $B(X, r)$ the open ball in this norm (of center $X$ and radius $r$ ). Define $k$-dimensional Hausdorff measure in $\mathbb{R}^{n}$, where $0<k \leq n$, as follows: For fixed $\delta>0$ and $E \subseteq \mathbb{R}^{n}$, consider all covers $L(\delta)=\left\{B\left(X_{i}, r_{i}\right)\right\}$ of $E$ such that $0<r_{i}<\delta$ for all $i$, and set

$$
\phi_{\delta}^{k}(E)=\inf _{L(\delta)} \sum \alpha(k) r_{i}^{k},
$$

where $\alpha(k)$ is the volume of the unit ball in $\mathbb{R}^{k}$. The $k$-dimensional Hausdorff measure of $E$ is then

$$
H^{k}(E)=\lim _{\delta \rightarrow 0} \phi_{\delta}^{k}(E)
$$

where $0<k \leq n$.

A domain is a connected open subset of $\mathbb{R}^{n}$. Suppose $D \subset \mathbb{R}^{n}, n \geq 2$, is a domain, that $x_{0} \in D$, and that $\mu$ is the harmonic measure for $D$ with respect to $x_{0}$, defined as usual by way of the Riesz representation theorem for continuous functions on $\partial D$ and a method of Perron, Wiener, and Brelot (see [Helms 1969]). In this paper we study the Hausdorff dimension of $\mu$, denoted by $\mathrm{H}-\operatorname{dim} \mu$ and defined as follows:

H-dim $\mu=\inf \left\{k\right.$ : there exists $E \subset \partial D$ with $H^{k}(E)=0$ and $\left.\mu(E)=1\right\}$.

Makarov [1985] showed that $\mathrm{H}-\operatorname{dim} \mu=1$ for a simply connected domain $D \subset \mathbb{R}^{2}$. Carleson [1985] showed that if $D=\mathbb{R}^{2} \backslash K$, where $K$ is a certain scale-invariant Cantor set, then H-dim $\mu \leq 1$. He was also the first to recognize the importance of $\int_{\partial D_{n}}\left|\nabla g_{n}\right| \log \left|\nabla g_{n}\right| d H^{1}$, where $g_{n}$ is the Green's function for $D_{n}$ with pole

MSC2000: 31B05, 31B15.

Keywords: harmonic measure, Green's function, Hausdorff measure, harmonic functions.

Lewis was partially supported by NSF grant 0139748 . Verchota was partially supported by NSF grant 9706648 . 
at $x_{0}$ and $\left(D_{n}\right)$ is an increasing sequence of smooth domains whose union is $D$. Jones and Wolff [1988] proved that H-dim $\mu \leq 1$ when $D \subset \mathbb{R}^{2}$ and $\mu$ exists. We also mention results of Batakis [1996], Kaufmann-Wu [1985], and Volberg [1993], who showed that

Hausdorff dimension of $\partial D=\inf \left\{k: H^{k}(\partial D)=0\right\}>\mathrm{H}-\operatorname{dim} \mu$

for certain fractal domains and domains whose complements are Cantor sets.

In higher dimensions $(n \geq 3)$ Bourgain [1987] showed that $\mu$ is absolutely continuous with respect to $H^{n-\varepsilon}$ on $\partial D$ for some $\varepsilon=\varepsilon(n)>0$ and any domain $D$ for which $\mu$ exists. Wolff [1995] used Carleson's idea and brilliant ideas of his own to construct what are now called Wolff snowflakes in $\mathbb{R}^{3}$, for which either $\mathrm{H}-\operatorname{dim} \mu>2$ or $\mathrm{H}-\operatorname{dim} \mu<2$. We outline his construction. Take the unit cube

$$
D_{0}=\left\{(x, t):\left|x_{1}\right|,\left|x_{2}\right|,|t| \leq \frac{1}{2}\right\}
$$

in $\mathbb{R}^{3}$ and decompose each face into Whitney squares whose side lengths are proportional to their distance from the edges of $\partial D_{0}$. Next one graphs above each face (i.e., in the normal direction to a face) a certain scaling and translation of a fixed piecewise linear and compactly supported function, say $\psi(y)=\psi\left(y_{1}, y_{2}\right)$, in such a way that the resulting graph function has support well inside each square. This can be done in a unique manner provided one first specifies what sides on each square are to correspond to the positive $y_{1}$ and $y_{2}$ axes under the scaling and translation. Let $D_{1}$ be the domain whose boundary is the union of the above surfaces. We say that $D_{1}$ is obtained from $D_{0}$ by adding blips to each subface. Next one divides the faces of $\partial D_{1}$ into Whitney squares whose side lengths are proportional to their distances from the edges of $\partial D_{1}$ and then repeats the above construction in each of the new Whitney squares, obtaining a domain $D_{2}$ for which $\partial D_{2}$ coincides with the new surface. Iterating this process gives $D=\bigcup_{m=1}^{\infty} D_{m}$. This construction is essentially unchanged in $\mathbb{R}^{n}$. Thus we assume $\psi: \mathbb{R}^{n-1} \rightarrow \mathbb{R}$ for some $n \geq 3$ so that $D \subset \mathbb{R}^{n}$. Finally let $\mu$ be harmonic measure for $D$ relative to $(0,0)$.

Put $D_{\infty}=\{(x, t): t>\psi(x)\}$. Let $g_{\infty}$ denote Green's function with pole at $\infty$, defined as the harmonic function in $D_{\infty}$ that is zero on $\partial D_{\infty}$ and equal to $t$ plus a bounded harmonic function in a neighborhood of $\infty$. The existence of $g_{\infty}$ follows from Schwarz reflection and the fact that $\psi$ has compact support.

Theorem A [Wolff 1995, p. 345]. Suppose $\psi$ has sufficiently small Lipschitz norm and set

$$
L=\int_{\partial D_{\infty}}\left|\nabla g_{\infty}\right| \log \left|\nabla g_{\infty}\right| d H^{n-1} .
$$

If $L<0$, the Wolff snowflake D obtained from $\psi$ by adding blips satisfies $H$-dim $\mu>$ $n-1$, while if $L>0$ then $H$-dim $\mu<n-1$. 
To prove this, Wolff used rate theorems for NTA domains to make estimates and applied an ergodic theorem of Shannon, McMillan and Breiman. His proof was for $n=3$, but it works unchanged for higher $n$.

It is not difficult to show that one can approximate each $C_{0}^{\infty}\left(\mathbb{R}^{n-1}\right)$ function $\psi$ on $\mathbb{R}^{n-1}$ by a sequence $\left(\psi_{n}\right)$ of piecewise linear functions with uniformly bounded supports in such a way that $\psi_{n} \rightarrow \psi$ as $n \rightarrow \infty$ uniformly in Lipschitz norm. Using results of Dahlberg (see [Wolff 1995, Lemma 2.11]), it follows easily that $L_{n}$ defined relative to $\psi_{n}$ converges to $L$ as $n \rightarrow \infty$. Thus to construct Wolff snowflakes where H-dim $\mu>n-1$ or $\mathrm{H}$-dim $\mu<n-1$ it suffices to find a $C_{0}^{\infty}\left(\mathbb{R}^{n-1}\right)$ function $\psi$ where $L<0$ or $L>0$, respectively, and approximate $\psi$ by a piecewise linear function. Thus we assume $\psi \in C_{0}^{\infty}\left(\mathbb{R}^{n-1}\right)$.

To continue, set $\phi(x, \varepsilon)=\varepsilon \psi(x)+\varepsilon^{2} \theta(x)$, where $\psi, \theta \in C_{0}^{\infty}\left(\mathbb{R}^{n-1}\right)$, and let $g(x, t, \varepsilon)$ be Green's function for $D(\varepsilon)=\left\{(x, t) \in \mathbb{R}^{n}: t>\phi(x, \varepsilon)\right\}$ with pole at $\infty$. Clearly $g(x, t, 0)=t$ and $D(0)=\{(x, t): t>0\}$. Put

$$
I(\varepsilon)=\int_{\partial D(\varepsilon)}|\nabla g(\cdot, \varepsilon)| \log |\nabla g(\cdot, \varepsilon)| d H^{n-1} .
$$

Using $g(x, \phi(x, \varepsilon), \varepsilon)=0$ on $\partial D(\varepsilon)$ and Schauder theory or just basic smoothness results about harmonic functions one sees that $I(\varepsilon)$ is infinitely differentiable. When $\theta \equiv 0$ Wolff shows that $I^{\prime}(0)=0, I^{\prime \prime}(0)=0$, and

$$
I^{\prime \prime \prime}(0)=\int_{\mathbb{R}^{n-1}}\left(\left(\frac{\partial \mathscr{P} \psi}{\partial t}\right)^{3}-3|\bar{\nabla} \psi|^{2} \frac{\partial \mathscr{P} \psi}{\partial t}\right) d x,
$$

where $\mathscr{P} \psi$ denotes the bounded harmonic extension of $\psi$ to $D(0), \nabla$ denotes the gradient in $x, t$, and $\bar{\nabla}$ denotes the gradient in $x$ only. See Section 3 for details.

Given a Wolff snowflake D, let $\tilde{\mu}$ be harmonic measure for $\mathbb{R}^{n} \backslash \bar{D}$ relative to a fixed point in $\mathbb{R}^{n} \backslash \bar{D}$. Using Taylor's theorem with remainder, one sees from (1-2) that if $I^{\prime \prime \prime}(0)<0$ one can approximate $\varepsilon \psi$, for $0<\varepsilon \leq \varepsilon_{0}$, by a piecewise linear function for which Wolff's construction gives a snowflake with $\mathrm{H}$-dim $\mu>n-1$ and H-dim $\tilde{\mu}<n-1$, while if $I^{\prime \prime \prime}(0)>0$ one gets a snowflake with H-dim $\mu<$ $n-1$ and $\mathrm{H}-\operatorname{dim} \tilde{\mu}>n-1$. Wolff obtained such examples for a piecewise linear approximation to a $C_{0}^{\infty}\left(\mathbb{R}^{n}\right)$ approximation of

$$
\psi(x)=-\frac{1}{\left(|x|^{2}+1\right)^{n / 2-1}}, \quad x \in \mathbb{R}^{n-1},
$$

when $n=3$. For this $\psi$ it is not difficult to show that $I^{\prime \prime \prime}(0)<0$ when $n \geq 3$. However for a given $\psi \in C_{0}^{\infty}\left(\mathbb{R}^{n-1}\right)$ and $n \geq 3$ the sign of the integral in (1-2) seemed rather mysterious (the integral is zero when $n=2$ by Cauchy's theorem). To shed some light on this problem, we have the following theorem, which is proved in Section 2.

Theorem 2. Let $\psi \in C_{0}^{\infty}\left(\mathbb{R}^{n-1}\right)$ be radial, nowhere positive, and nondecreasing as a function of $|x|$ on $[0, \infty)$. If $\psi \not \equiv 0$ and $\theta \equiv 0$, then $I^{\prime \prime \prime}(0)<0$. 
(Radial of course means that $\psi(x)=\psi(y)$ if $x=y$.)

To sum up once again, if $\psi$ is as in Theorem 2 and $\varepsilon>0$ is small enough, $\varepsilon \psi$ can be approximated arbitrarily closely in Lipschitz norm by a piecewise linear function in such a way that the corresponding Wolff snowflake has H-dim $\mu>n-1$ while $\mathrm{H}$-dim $\tilde{\mu}<n-1$. If $\varepsilon<0$ is small enough, the opposite inequalities hold. The proof is quite simple; unfortunately it was only after considerable effort that we conceived of the theorem and its proof. For a discussion of the motivation behind Theorem 2 and the efforts involved in its proof; see the Remark on page 144.

If $I^{\prime \prime \prime}(0)=0$ for given $\psi, \theta \in C_{0}^{\infty}\left(\mathbb{R}^{n-1}\right)$, the sign of $I^{\prime \prime \prime \prime}(0)$ becomes important in Wolff's construction. In this case it is remarked in [Wolff 1995, p. 363] that (again when $\theta \equiv 0$ ) if $I^{\prime \prime \prime \prime}(0)<0$, there is a piecewise linear function for which the corresponding Wolff snowflake satisfies $\mathrm{H}-\operatorname{dim} \mu>n-1$ and $\mathrm{H}-\operatorname{dim} \tilde{\mu}>n-1$. If $I^{\prime \prime \prime \prime}(0)>0$, the opposite inequalities hold for $\mathrm{H}-\operatorname{dim} \mu, \mathrm{H}-\operatorname{dim} \tilde{\mu}$. Wolff conjectures that there exist examples where the fourth derivative has either sign, although he notes that he has not computed $I^{\prime \prime \prime \prime}(0)$ and this derivative looks complicated. Thus he conjectures the existence of Wolff snowflakes in $\mathbb{R}^{n}, n \geq 3$, for which harmonic measure on both sides of the boundary can have Hausdorff dimension either $<n-1$ or $>n-1$. In Section 3 we compute the first four derivatives of $I$ at $\varepsilon=0$ relative to $\psi, \theta \in C_{0}^{\infty}\left(\mathbb{R}^{n-1}\right)$; see (3-20). In Section 4 we begin the proof of the following theorem:

Theorem 3. If $n \geq 3, \psi(x)=(2-n) x_{1}\left(|x|^{2}+1\right)^{-n / 2}, x \in \mathbb{R}^{n-1}, \theta \equiv 0$ and

$$
A=\frac{3 n \pi^{n / 2}(n-2)^{4} \Gamma\left(\frac{3 n}{2}+\frac{1}{2}\right) 2^{-2 n-1}}{\Gamma(n) \Gamma\left(n+\frac{1}{2}\right)(n+1)}
$$

then $I^{\prime \prime \prime}(0)=0$ and

$$
\begin{aligned}
& A^{-1} I^{\prime \prime \prime \prime}(0) \\
& =\left(\frac{-1}{16} \frac{\Gamma\left(n+\frac{1}{2}\right) \Gamma\left(n-\frac{1}{2}\right) \Gamma\left(\frac{n}{2}-1\right) \Gamma\left(\frac{3 n}{2}+1\right)}{\Gamma(n+1)^{2} \Gamma\left(\frac{n}{2}-\frac{1}{2}\right) \Gamma\left(\frac{3 n}{2}+\frac{1}{2}\right)}\right. \\
& \left.\quad \times \frac{n\left(16+16 n-72 n^{2}-44 n^{3}-11 n^{4}+12 n^{6}\right)}{(n+1)^{2}(n-1)}+\frac{3 n^{3}+2 n^{2}+7 n+2}{2 n+1}\right)>0 .
\end{aligned}
$$

Here $I^{\prime \prime \prime}(0), I^{\prime \prime \prime \prime}(0)$ denote the integrals in $(1-2)$ and $(3-20)$ rather than the third and fourth derivatives of $I(\varepsilon)$ in $(1-1)$ (defined relative to the above $\psi$ ) since for derivatives it is necessary that $\psi \in C_{0}^{\infty}\left(\mathbb{R}^{n-1}\right)$. Let $\psi_{m}=\phi_{m} \psi$, where $0 \leq \phi_{m} \in C_{0}^{\infty}\left(\mathbb{R}^{n-1}\right)$ is a radial bump function with $\phi_{m}(x)=1$ when $|x|<m$ and $\phi_{m}(x)=0$ when $|x|>2 \mathrm{~m}$. Also $\psi$ is as in Theorem 3. We note that the integral in (1-2) corresponding to $\psi_{m}$ is still zero. Indeed $\psi_{m}$ is an odd function of $x_{1}$, so using difference quotients we deduce first that $(\partial / \partial t) \mathscr{P} \psi_{m}$ is odd in $x_{1}$ and then that the integrand in (1-2) is odd in $x_{1}$. Thus our claim is true. Also, choosing $\left(\phi_{m}\right)$ appropriately, one sees from Hardy space theory (see [Stein 1970, 
Chapter 7]) that the integral in (3-20) defining $I^{\prime \prime \prime \prime}(0)$ relative to $\psi_{m}$ converges to the corresponding integral defined relative to $\psi$ as $m \rightarrow \infty$. Thus there exists a $C_{0}^{\infty}\left(\mathbb{R}^{n-1}\right)$ approximation to $\psi$ in Theorem 3 for which $I^{\prime \prime \prime}(0)=0$ and $I^{\prime \prime \prime \prime}(0)>0$. We can now argue as in [Wolff 1995] to get:

Corollary 1. There exist Wolff snowflakes in $\mathbb{R}^{n}, n \geq 3$, with $H$-dim $\mu<n-1$ and $H$-dim $\tilde{\mu}<n-1$.

We do not know whether there are examples in $\mathbb{R}^{n}$ of graph domains $D(\varepsilon)$ with $\psi \in C_{0}^{\infty}\left(\mathbb{R}^{n-1}\right), \theta \equiv 0, I^{\prime \prime \prime}(0)=0, I^{\prime \prime \prime \prime}(0)<0$. We tried numerous examples in $\mathbb{R}^{4}$ and all gave positive fourth derivative (see Section 6). However, by considering more general graph domains we can answer Wolff's main question. We prove the following in Section 6:

Theorem 4. If $\psi$ is as in Theorem 3 and $\theta=\psi \psi_{t}$, then $I^{\prime \prime \prime}(0)=0$ and

$$
I^{\prime \prime \prime \prime}(0)=-\frac{3 \pi^{n / 2} \Gamma\left(\frac{3 n}{2}+\frac{1}{2}\right)(n-2)^{4} n^{2}\left(3 n^{3}+2 n^{2}-13 n-6\right)}{\Gamma\left(n+\frac{1}{2}\right) \Gamma(n) 2^{2 n+1}(n+1)(2 n+3)(2 n+1)}<0 .
$$

We note that $I^{\prime \prime \prime}(0)$ (see (3-14)) is independent of the choice of $\theta$. Using this fact and repeating the argument after Theorem 3 (but approximating $\varepsilon \psi+\varepsilon^{2} \theta$ instead) we obtain:

Corollary 2. There exist Wolff snowflakes in $\mathbb{R}^{n}, n \geq 3$, with $H$-dim $\mu>n-1$ and $H$-dim $\tilde{\mu}>n-1$.

In view of Corollaries 1 and 2 we conclude that Wolff's main conjecture is valid. Finally we mention that in Section 6 we shall make three conjectures related to Theorems 3, 4 and Corollaries 1, 2, as well as discuss some examples in $\mathbb{R}^{4}$ where the first two conjectures are valid.

\section{Proof of Theorem 2}

To lighten the notation we write $\psi=\psi(x, t)$ for the bounded harmonic extension $\mathscr{P} \psi(x, t)$ of $\psi(x)$ to $D(0)=\{(x, t): t>0\}$. Thus $\psi(x, 0)$ satisfies the hypotheses of Theorem 2 and we can write $\psi(r, t)$ for $\psi(x, t)$, where $r=|x|$. Note that $\psi$ satisfies Laplace's equation when $t>0$, so in cylindrical coordinates

$$
r^{2-n}\left(r^{n-2} \psi_{r}\right)_{r}+\psi_{t t}=0 .
$$

We shall need the following facts:

$$
\begin{aligned}
\lim _{r \rightarrow 0} \psi_{r}(r, t)=0 & \text { for } 0 \leq t<\infty, \\
\psi_{r}(r, t) \geq 0 & \text { when } r, t>0 .
\end{aligned}
$$

Equation (2-2) can be seen using the fact that $\psi(|x|, t)$ is infinitely differentiable at $x=0$ when $t \geq 0$. One can also prove it, for $t>0$, by differentiating under the integral sign in the Poisson integral formula for $\psi$ and taking a limit as $r \rightarrow 0$. As 
for $(2-3)$, assume the contrary. From $(2-2)$, the fact that $\psi(r, 0)$ is nondecreasing on $[0, \infty)$ and the fact that $\psi$ is infinitely differentiable in $\bar{D}(0)$ (as follows from Schauder theory) we deduce that $\psi_{r}$ has a negative minimum (absolute) for some $(r, t)$ with $r>0, t>0$. At this value of $(r, t)$ we have $\psi_{r r}=0$ and $\psi_{r r r}+\psi_{r t t} \geq 0$, by the second derivative test from elementary calculus. Now (2-1) can be written as $\psi_{r r}+(n-2) r^{-1} \psi_{r}+\psi_{t t}=0$. Differentiating this with respect to $r$ we get $\psi_{r r r}+(n-2) \psi_{r r} r^{-1}-(n-2) r^{-2} \psi_{r}+\psi_{r t t}=0$, which leads to a contradiction, since from the remark above this expression must be positive. Thus (2-3) is valid.

Now if $\Lambda=(0, \infty) \times(0, \infty)$, we have

$$
\begin{aligned}
\int_{0}^{\infty}\left(\psi_{t}^{3}-3 \psi_{r}^{2} \psi_{t}\right)(r, 0) r^{n-2} d r & =\int_{\Lambda} \frac{d}{d t}\left(-\psi_{t}^{3}+3 \psi_{r}^{2} \psi_{t}\right) r^{n-2} d r d t \\
& =\int_{\Lambda}\left(-3 \psi_{t}^{2} \psi_{t t}+6 \psi_{r} \psi_{t} \psi_{t r}\right) r^{n-2} d r d t \\
& =I_{1}+I_{2} .3 \int_{\Lambda} \psi_{r}^{2} \psi_{t t} r^{n-2} d r d t
\end{aligned}
$$

To handle $I_{1}$ in (2-4) we use (2-1) and integrate by parts in $r$ holding $t$ fixed. We get

$$
I_{1}=\int_{\Lambda} 3 \psi_{t}^{2}\left(r^{n-2} \psi_{r}\right)_{r} d r d t+\int_{\Lambda} 6 \psi_{r} \psi_{t} \psi_{t r} r^{n-2} d r d t=0
$$

As for $I_{2}$ we again use (2-1) and write

$$
\begin{aligned}
I_{2} & =-3 \int_{\Lambda} \psi_{r}^{2}\left(r^{n-2} \psi_{r}\right)_{r} d r d t \\
& =-\int_{\Lambda}\left(r^{n-2} \psi_{r}^{3}\right)_{r} d r d t-2(n-2) \int_{\Lambda} r^{n-3} \psi_{r}^{3} d r d t<0,
\end{aligned}
$$

since the first integral is zero, as follows from integrating in $r$ holding $t$ fixed, while the second integral is negative, as follows from (2-2) and our assumption that $\psi \not \equiv 0$. In obtaining (2-5) and (2-6) we have integrated by parts numerous times, assuming $\psi$ and its derivatives have rapid enough decay at $\infty$ to insure there are no boundary terms. This is justified, as we see from simple estimates on the Poisson integral of $\psi$ and its derivatives, in view of the fact that $\psi(\cdot, 0) \in C_{0}^{\infty}\left(\mathbb{R}^{n-1}\right)$. Using (2-5), (2-6) in $(2-4)$ we conclude that $I^{\prime \prime \prime}(0)<0$. The proof of Theorem 2 is complete.

Remark. Our interest in Theorem 2 stems from work in [Lewis and Vogel 1991; 2001], where blips were added to a ball in such a way as to produce an example (for $n \geq 3$ ) of a bounded domain $D \subset \mathbb{R}^{n}$ distinct from the ball and satisfying $\mu=H^{n-1}$ on $\partial D$, where $\mu$ is harmonic measure defined relative to $0 \in D$. In the first of these papers we showed that $D$ can be chosen homeomorphic to a ball, while the second improved on this result by showing that $D$ can be the image of a ball under a quasiconformal mapping of $\mathbb{R}^{n}$. In this construction the sign of the integral in (1-1) 
was crucial. In [Lewis and Vogel 1991] certain multiples, scalings, translations, and $C_{0}^{\infty}\left(\mathbb{R}^{n-1}\right)$ approximations of the function in (1-3) (restricted to $\mathbb{R}^{n-1}$ ) were used to construct our blips, while in [Lewis and Vogel 2001] it was necessary for the construction of our quasiconformal mapping to add spherical blips. As a consequence we had to prove Theorem 2 when $\psi(x)=-\max \left(1-|x|^{2}, 0\right)$ on $\mathbb{R}^{n-1}$. To appreciate the simple proof in Theorem 2 we briefly outline our efforts to prove the theorem for this $\psi$.

We first used Maple to obtain strong evidence that $I^{\prime \prime \prime}(0)<0$ when $n=2,4,6,8$, and 10. Then using separation of variables and integrals of Bessel functions we found a representation of $\psi_{t}$ via hypergeometric functions. Let $\Gamma$ be the Euler gamma function. If $r=|x|$, then for $0<r<1$,

$$
-\psi_{t}(r, 0)=-c_{n} F\left(\frac{n}{2},-\frac{1}{2}, \frac{n}{2}-\frac{1}{2}, r^{2}\right),
$$

where $F(a, b, c, z)$ is the hypergeometric function and

$$
c_{n}=\frac{2 \Gamma\left(\frac{n}{2}\right)}{\Gamma\left(\frac{n}{2}-\frac{1}{2}\right) \Gamma\left(\frac{3}{2}\right)} .
$$

Using properties of hypergeometric functions we found many nice formulas involving $\psi_{t}$. For example, if $n=2 k$, where $k \geq 2$ is an integer, $\psi_{t}(r, 0)$ equals

$$
\frac{2(-1)^{k-2}}{\pi(k-1) !\left(1-r^{2}\right)} \frac{d^{k-2}}{d\left(r^{2}\right)^{k-2}}\left(\left(1-r^{2}\right)^{k-1}\left(\ln \left(\frac{1+r}{1-r}\right)\left(\frac{1}{2 r}-\frac{3 r}{2}\right)+3\right)\right)
$$

whenever $0<r<1$. However none of our formulas were of much help in calculating the integral in (1-2).

Finally we tried asymptotic estimates as $n \rightarrow \infty$ of $I^{\prime \prime \prime}(0)$ using

$$
-F\left(\frac{n}{2},-\frac{1}{2}, \frac{n}{2}-\frac{1}{2}, r^{2}\right) \geq-1+\frac{n}{n-1}\left(1-\left(1-r^{2}\right)^{1 / 2}\right) .
$$

From this estimate and Stirling's formula we found after a lot of work that $I^{\prime \prime \prime}(0)<$ 0 for $n \geq 10$. Based on this example, we conjectured that if $-\psi$ is radial, positive, and smooth on $\mathbb{R}^{n-1}$ with compact support, then $I^{\prime \prime \prime}(0)<0$. This was disproved with the following example. For fixed $(x, t) \in \mathbb{R}^{4}$ and $0<a, a \neq 1$, let

$$
-\psi(x, t)=\frac{1}{|x|^{2}+(t+1)^{2}}-\frac{a^{2}}{|x|^{2}+(t+a)^{2}} .
$$

Clearly $\psi$ is harmonic in $\mathbb{R}^{n} \backslash\{(0,-1),(0,-a)\}$ and $\psi(x, 0)$ is radial. Also for $0<$ $a<1$ one can show that $-\psi(x, 0)>0$ and that the corresponding integral in (1-2) is 0 . Although $\psi(x, 0)$ does not have compact support it turns out that for a given $a$ this function can be approximated by an infinitely differentiable nonnegative radial function with compact support in such a way that the integral in (1-2) remains zero (see Section 6 for more discussion of this example). Reworking the statement above, in light of this example, we formulated Theorem 2. The simple proof was only found after trying several more complicated arguments. 


\section{The fourth derivative}

In this section we find the fourth derivative of $I(\varepsilon)$ at $\varepsilon=0$ (defined as in (1-1) for $\left.\psi, \theta \in C_{0}^{\infty}\left(\mathbb{R}^{n-1}\right)\right)$. To begin, as in Section 1 , put $\phi(x, \varepsilon)=\varepsilon \psi(x)+\varepsilon^{2} \theta(x)$, set $D(\varepsilon)=\{(x, t): t>\phi(x, \varepsilon)\}$ for $\varepsilon \in[-1,1]$, and let $g(\cdot, \varepsilon)$ be the corresponding Green's function for $D(\varepsilon)$ with pole at $\infty$. Following [Wolff 1995, pp. 360-362] we observe from Schauder-type estimates that $g(\cdot, \varepsilon)$ is infinitely differentiable in $\bar{D}(\varepsilon) \times[-1,1]$ as a function of $(x, \varepsilon)$. Also, since $g(x, \phi(x, \varepsilon), \varepsilon) \equiv 0$ when $x \in \mathbb{R}^{n-1}$, we get from differentiating with respect to $x_{i}, 1 \leq i \leq n-1$, that

$$
\bar{\nabla} g(x, \phi(x, \varepsilon), \varepsilon)=-g_{t}(x, \phi(x, \varepsilon), \varepsilon)\left(\varepsilon \bar{\nabla} \psi(x)+\varepsilon^{2} \bar{\nabla} \theta(x)\right),
$$

where once again $\bar{\nabla}$ denotes the gradient in $x$ only. Differentiating with respect to $\varepsilon$ and using the chain rule gives, for all $x \in \mathbb{R}^{n-1}$ and $\varepsilon \in[-1,1]$,

$$
g_{\varepsilon}(x, \phi(x, \varepsilon), \varepsilon)+g_{t}(x, \phi(x, \varepsilon), \varepsilon)(\psi(x)+2 \varepsilon \theta(x))=0,
$$

where $g_{\varepsilon}(x, t, \varepsilon)$ denotes the partial of $g(x, t, \varepsilon)$ with respect to $\varepsilon$. Evaluating (3-2) at $\varepsilon=0$ yields

$$
g_{\varepsilon}(x, 0,0)=-g_{t}(x, 0,0) \psi(x)=-\psi(x),
$$

since $g(x, t, 0)=t$. Furthermore since $g_{\varepsilon}(x, t, \varepsilon)$ is harmonic in $(x, t)$ we see that $g_{\varepsilon}(x, t, 0)=-\mathscr{P} \psi(x, t)$, where $\mathscr{P} \psi$ as defined earlier denotes the bounded harmonic extension of $\psi$ to $D(0)$, that is, the Poisson integral of $\psi$. As in Section 2 we write $\psi(x, t)$ for $\mathscr{P} \psi(x, t)$, and likewise $\theta(x, t)$ for $\mathscr{P} \theta(x, t)$. Taking another derivative with respect to $\varepsilon$ in (3-2) gives, for all $x \in \mathbb{R}^{n-1}$ and $\varepsilon \in[-1,1]$,

$$
\begin{aligned}
g_{t t}(x, \phi(x, \varepsilon), \varepsilon)(\psi(x)+2 \varepsilon \theta(x))^{2} & \\
+2 g_{t \varepsilon}(x, \phi(x, \varepsilon), \varepsilon)(\psi(x)+2 \varepsilon \theta(x)) & +g_{\varepsilon \varepsilon}(x, \phi(x, \varepsilon), \varepsilon) \\
& +2 \theta(x) g_{t}(x, \phi(x, \varepsilon), \varepsilon)=0 .
\end{aligned}
$$

Evaluating at $\varepsilon=0$ yields

$$
g_{\varepsilon \varepsilon}(x, 0,0)=2 \psi(x) \psi_{t}(x, 0)-2 \theta(x), \quad x \in \mathbb{R}^{n-1} .
$$

From this equality and the harmonicity of $g_{\varepsilon \varepsilon}$ we conclude that

$$
g_{\varepsilon \varepsilon}(x, t, 0)=2 \mathscr{P}\left(\psi \psi_{t}-\theta\right)(x, t), \quad(x, t) \in D(0) .
$$

Next observe that

$$
I(\varepsilon)=\int_{\mathbb{R}^{n-1}}|\nabla g(x, \phi(x, \varepsilon), \varepsilon)| \log |\nabla g(x, \phi(x, \varepsilon), \varepsilon)| \sqrt{1+|\bar{\nabla} \phi(x, \varepsilon)|^{2}} d x .
$$

Also, $(-\bar{\nabla} \phi(x, \varepsilon), 1)\left(1+|\bar{\nabla} \phi|^{2}\right)^{-1 / 2}$ is the inner normal to $\partial D(\varepsilon)$ at $(x, \phi(x, \varepsilon))$ and $\nabla g(x, \phi(x, \varepsilon), \varepsilon)$ is parallel to this inner normal. Thus at $(x, \phi(x, \varepsilon))$ we have

$$
|\nabla g|=\frac{-\langle\bar{\nabla} \phi, \bar{\nabla} g\rangle+g_{t}}{\sqrt{1+|\bar{\nabla} \phi|^{2}}},
$$


where $\langle\cdot, \cdot\rangle$ denotes the usual inner product on $\mathbb{R}^{n-1}$. Substituting this expression into the integrand in (3-7), canceling the square roots, and taking logarithms we obtain

$$
\left(-\langle\bar{\nabla} g, \bar{\nabla} \phi\rangle+g_{t}\right)\left(\log \left(-\langle\bar{\nabla} g, \bar{\nabla} \phi\rangle+g_{t}\right)-\frac{1}{2} \log \left(1+|\bar{\nabla} \phi(x, \varepsilon)|^{2}\right)\right) .
$$

Using (3-1) and the above equality the integrand in (3-7) now becomes

$$
\left(1+|\bar{\nabla} \phi(x, \varepsilon)|^{2}\right) g_{t}\left(\log \left(g_{t}\right)+\frac{1}{2} \log \left(1+|\bar{\nabla} \phi(x, \varepsilon)|^{2}\right)\right)
$$

at $(x, \phi(x, \varepsilon)), x \in \mathbb{R}^{n-1}$ : We expand this expression in $\varepsilon$ at 0 through powers of $\varepsilon^{4}$, since our goal is to find $I^{\prime \prime \prime \prime}(0)$. For this purpose let

$$
g_{t}(x, \phi(x, \varepsilon), \varepsilon)=1+a_{1} \varepsilon+a_{2} \varepsilon^{2}+a_{3} \varepsilon^{3}+a_{4} \varepsilon^{4}+\cdots
$$

and set

$$
w=a_{1} \varepsilon+a_{2} \varepsilon^{2}+a_{3} \varepsilon^{3}+a_{4} \varepsilon^{4}+\cdots .
$$

The logarithm of $g_{t}$ then becomes $w-\frac{1}{2} w^{2}+\frac{1}{3} w^{3}-\frac{1}{4} w^{4}+\cdots$. Also,

$$
|\bar{\nabla} \phi(x, \varepsilon)|^{2}=\varepsilon^{2}|\bar{\nabla} \psi|^{2}(x)+2 \varepsilon^{3}|\bar{\nabla} \psi(x), \bar{\nabla} \theta(x)\rangle+\varepsilon^{4}|\bar{\nabla} \theta(x)|^{2}
$$

and

$$
\begin{aligned}
\log \left(1+|\bar{\nabla} \phi(x, \varepsilon)|^{2}\right) & =|\bar{\nabla} \phi(x, \varepsilon)|^{2}-\frac{1}{2}\left(|\bar{\nabla} \phi(x, \varepsilon)|^{2}\right)^{2}+\cdots \\
& =\varepsilon^{2}|\bar{\nabla} \psi(x)|^{2}+2 \varepsilon^{3}|\bar{\nabla} \psi(x), \bar{\nabla} \theta(x)\rangle+\varepsilon^{4}\left(|\bar{\nabla} \theta(x)|^{2}\right. \\
& \left.\quad-\frac{1}{2}|\bar{\nabla} \psi(x)|^{4}\right)+\cdots
\end{aligned}
$$

Using these equalities, multiplying out the expression above and collecting powers of $\varepsilon$ up to order 4 we have for the integrand in (3-7)

$$
\begin{aligned}
\varepsilon a_{1}+ & \frac{1}{2} \varepsilon^{2}\left(2 a_{2}+a_{1}^{2}+|\bar{\nabla} \psi|^{2}\right) \\
+ & \frac{1}{6} \varepsilon^{3}\left(6 a_{3}+6 a_{1} a_{2}-a_{1}^{3}+9 a_{1}|\bar{\nabla} \psi|^{2}+6\langle\bar{\nabla} \psi, \bar{\nabla} \theta\rangle\right) \\
+ & \frac{1}{24} \varepsilon^{4}\left(24 a_{4}+24 a_{1} a_{3}+\left(36|\bar{\nabla} \psi|^{2}-12 a_{1}^{2}+12 a_{2}\right) a_{2}\right. \\
& \left.\quad+\left(2 a_{1}^{2}+12|\bar{\nabla} \psi|^{2}\right) a_{1}^{2}+6|\bar{\nabla} \psi|^{4}+12|\bar{\nabla} \theta|^{2}+72\langle\bar{\nabla} \psi, \bar{\nabla} \theta\rangle a_{1}\right) \\
& +O\left(\varepsilon^{5}\right),
\end{aligned}
$$

where the last term denotes a bounded term in $\varepsilon^{5}$ on $[-1,1]$. From Taylor's theorem and the smoothness of $g$ and its derivatives on $\bar{D}(\varepsilon)$, we deduce that $k ! a_{k}=$ $k ! a_{k}(x)$ is the $k$-th derivative of $g_{t}(x, \phi(x, \varepsilon), t)$ evaluated at $\varepsilon=0$. This $k$-th total derivative in $\varepsilon$ is easily seen to be equal to the operator $(\psi+2 \varepsilon \theta)(\partial / \partial t)+(\partial / \partial \varepsilon)$ applied $k$ times to $g_{t}$ at $(x, \phi(x, \varepsilon))$. Using this fact and (3-3) we deduce at $x \in \mathbb{R}^{n-1}$ and $\varepsilon=0$ that

$$
a_{1}=g_{\varepsilon t}=-\psi_{t} .
$$


From (3-7)-(3-9) we conclude that

$$
I^{\prime}(0)=\int_{\mathbb{R}^{n-1}} a_{1}=-\int_{\mathbb{R}^{n-1}} \psi_{t}(x, 0) d x=0,
$$

as follows from Green's first identity (the integral is the normal derivative of a harmonic function) using smoothness of derivatives of $\psi_{t}$ in $\bar{D}(0)$ and the fact that $\partial^{k} \psi_{t}(x, t)=O\left(\left(|x|^{2}+t^{2}\right)^{-(n+k) / 2}\right)$ in a neighborhood of $\infty$. Here $\partial^{k}$ denotes an arbitrary $k$-th partial of $\psi_{t}$ in either $x$ or $t$. Also, using the harmonicity of $g_{\varepsilon}$ and (3-3) we get at $x \in \mathbb{R}^{n-1}$ and $t=0=\varepsilon$ the relation

$$
2 a_{2}=\psi^{2} g_{t^{3}}+2 \theta g_{t^{2}}+2 \psi g_{\varepsilon t^{2}}+g_{\varepsilon^{2} t}=2 \psi \bar{\Delta} \psi+g_{\varepsilon^{2} t} .
$$

Using (3-11), (3-7)-(3-9), Green's first identity and integrating by parts we find, in view of the above decay estimates,

$$
\begin{aligned}
I^{\prime \prime}(0) & =\int_{\mathbb{R}^{n-1}}\left(a_{1}^{2}+2 a_{2}+|\bar{\nabla} \psi|^{2}\right) \\
& =\int_{\mathbb{R}^{n-1}}\left(\psi_{t}^{2}+2 \psi \bar{\Delta} \psi+g_{\varepsilon^{2} t}+|\bar{\nabla} \psi|^{2}\right) d x \\
& =\int_{\mathbb{R}^{n-1}}\left(\psi_{t}^{2}-|\bar{\nabla} \psi|^{2}\right) d x=0 .
\end{aligned}
$$

The last integral is zero from a Rellich-type identity; see [Stein 1970, Chapter 7]. Next we note from (3-3) that at $x \in \mathbb{R}^{n-1}$ and $t=0=\varepsilon$,

$$
\begin{aligned}
6 a_{3}=\psi^{3} g_{t^{4}}+6 \psi \theta g_{t^{3}}+3 \psi^{2} g_{\varepsilon t^{3}} & +3 \psi g_{\varepsilon^{2} t^{2}}+g_{\varepsilon^{3} t}+6 \theta g_{\varepsilon t^{2}} \\
& =3 \psi^{2} \bar{\Delta} \psi_{t}-3 \psi \bar{\Delta} g_{\varepsilon^{2}}+g_{\varepsilon^{3} t}+6 \theta \bar{\Delta} \psi
\end{aligned}
$$

Using (3-13), (3-11), (3-7)-(3-9), integrating by parts using the harmonicity of derivatives of $g_{t}$, as well as Green's first and second identities (to handle the terms in $g_{\varepsilon^{3} t}$ and $g_{\varepsilon^{2} t}$ ), we conclude that

$$
\begin{aligned}
I^{\prime \prime \prime}(0)= & \int_{\mathbb{R}^{n-1}}\left(6 a_{3}+6 a_{1} a_{2}-a_{1}^{3}+9 a_{1}|\bar{\nabla} \psi|^{2}+6\langle\bar{\nabla} \psi, \bar{\nabla} \theta\rangle\right) d x \\
= & \int_{\mathbb{R}^{n-1}}\left(3 \psi^{2} \bar{\Delta} \psi_{t}-3 \psi \bar{\Delta} g_{\varepsilon^{2}}+g_{\varepsilon^{3} t}-3 \psi_{t}\left(2 \psi \bar{\Delta} \psi+g_{\varepsilon^{2} t}\right)\right. \\
& \left.+\psi_{t}^{3}-9 \psi_{t}|\bar{\nabla} \psi|^{2}+6 \theta \bar{\Delta} \psi+6\langle\bar{\nabla} \psi, \bar{\nabla} \theta\rangle\right) d x \\
= & \int_{\mathbb{R}^{n-1}}\left(\psi_{t}^{3}-3 \psi_{t}|\bar{\nabla} \psi|^{2}\right) d x .
\end{aligned}
$$

We note that the derivatives in (3-10), (3-12), (3-14) agree with those in [Wolff 1995] even when $\theta \not \equiv 0$. Finally observe for $x \in \mathbb{R}^{n-1}, t=0=\varepsilon$, that

$$
\begin{aligned}
& 24 a_{4}= \psi^{4} g_{t^{5}}+4 \psi^{3} g_{\varepsilon t^{4}}+12 \theta \psi^{2} g_{t^{4}}+12 \theta^{2} g_{t^{3}}+ \\
& 6 \psi^{2} g_{\varepsilon^{2} t^{3}}+4 \psi g_{\varepsilon^{3} t^{2}}+g_{\varepsilon^{4} t}+24 \theta \psi g_{\varepsilon t^{3}}+12 \theta g_{t^{2} \varepsilon^{2}} \\
&=-4 \psi^{3} \bar{\Delta} \bar{\Delta} \psi-6 \psi^{2} \bar{\Delta} g_{\varepsilon^{2} t}+4 \psi g_{\varepsilon^{3} t^{2}}+g_{\varepsilon^{4} t}+24 \theta \psi \bar{\Delta} \psi_{t}-12 \theta \bar{\Delta} g_{\varepsilon^{2}} .
\end{aligned}
$$


Using (3-15), (3-13), (3-11), (3-7)-(3-9) we deduce that at $x \in \mathbb{R}^{n-1}, \varepsilon=0$ we have

$$
\begin{aligned}
& I^{\prime \prime \prime \prime}(0)=\int_{\mathbb{R}^{n-1}}\left(24 a_{4}+24 a_{1} a_{3}+\left(36|\bar{\nabla} \psi|^{2}-12 a_{1}^{2}+12 a_{2}\right) a_{2}+\left(2 a_{1}^{2}+12|\bar{\nabla} \psi|^{2}\right) a_{1}^{2}\right. \\
& \left.+6|\bar{\nabla} \psi|^{4}+12|\bar{\nabla} \theta|^{2}-72\langle\bar{\nabla} \psi, \bar{\nabla} \theta\rangle \psi_{t}\right) d x \\
& =\int_{\mathbb{R}^{n-1}}\left(-4 \psi^{3} \bar{\Delta} \bar{\Delta} \psi-6 \psi^{2} \bar{\Delta} g_{\varepsilon^{2} t}+4 \psi g_{\varepsilon^{3} t^{2}}+g_{\varepsilon^{4} t}+24 \theta \psi \bar{\Delta} \psi_{t}-12 \theta \bar{\Delta} g_{\varepsilon^{2}}\right. \\
& -4 \psi_{t}\left(3 \psi^{2} \bar{\Delta} \psi_{t}-3 \psi \bar{\Delta} g_{\varepsilon^{2}}+g_{\varepsilon^{3} t}+6 \theta \bar{\Delta} \psi\right) \\
& +\left(18|\bar{\nabla} \psi|^{2}-6 \psi_{t}^{2}+3\left(2 \psi \bar{\Delta} \psi+g_{\varepsilon^{2} t}\right)\right)\left(2 \psi \bar{\Delta} \psi+g_{\varepsilon^{2} t}\right) \\
& \left.+2 \psi_{t}^{4}+12|\bar{\nabla} \psi|^{2} \psi_{t}^{2}+6|\bar{\nabla} \psi|^{4}+12|\bar{\nabla} \theta|^{2}-72\langle\bar{\nabla} \psi, \bar{\nabla} \theta\rangle \psi_{t}\right) d x \text {. }
\end{aligned}
$$

Using Green's first identity we see that the term in $g_{\varepsilon^{4} t}$ integrates to zero. Also using Green's second identity we deduce that the terms in $g_{\varepsilon^{3} t^{2}}$ and $g_{\varepsilon^{3} t}$ taken together integrate to zero. Thus the only terms left involve at most two partials in $\varepsilon$. As for these terms we observe that

$$
\begin{aligned}
\int_{\mathbb{R}^{n-1}}\left(\left(12 \psi \bar{\Delta} \psi+18|\bar{\nabla} \psi|^{2}-6 \psi_{t}^{2}\right) g_{\varepsilon^{2} t}\right. & \left.-6 \psi^{2} \bar{\Delta} g_{\varepsilon^{2} t}\right) d x \\
& =\int_{\mathbb{R}^{n-1}} 6\left(|\bar{\nabla} \psi|^{2}-\psi_{t}^{2}\right) g_{\varepsilon^{2} t} d x
\end{aligned}
$$

Also combining the two terms in $\bar{\Delta} g_{\varepsilon^{2}}$ we see from (3-5) that the sum can be written as $6 g_{\varepsilon^{2}} \bar{\Delta} g_{\varepsilon^{2}}$. Using this fact, (3-5), integration by parts, and the Rellichtype formula mentioned after $(3-12)$ we see that

$$
\begin{aligned}
& \int_{\mathbb{R}^{n-1}}\left(6 g_{\varepsilon^{2}} \bar{\Delta} g_{\varepsilon^{2}}+3\left(g_{\varepsilon^{2} t}\right)^{2}\right) d x \\
& =\int_{\mathbb{R}^{n-1}}\left(-6\left|\bar{\nabla} g_{\varepsilon^{2}}\right|^{2}+3\left(g_{\varepsilon^{2} t}\right)^{2}\right) d x=\int_{\mathbb{R}^{n-1}}-3\left|\bar{\nabla} g_{\varepsilon^{2}}\right|^{2} d x \\
& =-12 \int_{\mathbb{R}^{n-1}}\left(\psi^{2}\left|\bar{\nabla} \psi_{t}\right|^{2}+\psi_{t}^{2}|\bar{\nabla} \psi|^{2}+2 \psi \psi_{t}\left\langle\bar{\nabla} \psi, \bar{\nabla} \psi_{t}\right\rangle\right. \\
& \left.\quad+|\bar{\nabla} \theta|^{2}-2\langle\bar{\nabla} \psi, \bar{\nabla} \theta\rangle \psi_{t}-2 \psi\left\langle\bar{\nabla} \psi_{t}, \bar{\nabla} \theta\right\rangle\right) d x
\end{aligned}
$$

Next note that

(3-19) $\int_{\mathbb{R}^{n-1}}\left(-4 \psi^{3} \bar{\Delta} \bar{\Delta} \psi+12 \psi^{2}(\bar{\Delta} \psi)^{2}\right) d x=\int_{\mathbb{R}^{n-1}}-24 \psi|\bar{\nabla} \psi|^{2} \bar{\Delta} \psi d x$.

Using (3-17)-(3-19) in (3-16) we get

(3-20) $\quad I^{\prime \prime \prime \prime \prime}(0)=\int_{\mathbb{R}^{n-1}}\left(12 \psi|\bar{\nabla} \psi|^{2} \bar{\Delta} \psi+24 \psi \psi_{t}\left\langle\bar{\nabla} \psi, \bar{\nabla} \psi_{t}\right\rangle+6\left(|\bar{\nabla} \psi|^{2}-\psi_{t}^{2}\right) g_{\varepsilon^{2} t}\right.$

$$
\left.+2 \psi_{t}^{4}+12|\bar{\nabla} \psi|^{2} \psi_{t}^{2}+6|\bar{\nabla} \psi|^{4}-24\langle\bar{\nabla} \psi, \bar{\nabla} \theta\rangle \psi_{t}\right) d x
$$

which we use for computations in the next two sections. 


\section{The bad integral}

Let $h(x, t)=\left(|x|^{2}+(t+1)^{2}\right)^{1-n / 2}$ be the fundamental solution to Laplace's equation in $\mathbb{R}^{n}$ with pole at $(0,-1)$ and recall that $\theta \equiv 0$, while

$$
\psi(x, t)=\frac{\partial h(x, t)}{\partial x_{1}}=\frac{(2-n) x_{1}}{\left(|x|^{2}+(t+1)^{2}\right)^{n / 2}}
$$

is the function in Theorem 3 when $t=0$. Also as pointed out in Section 1, the integrand in (1-2) or (3-14) for this $\psi$ is odd in $x_{1}$. Thus, $I^{\prime \prime \prime}(0)=0$. In this section we begin the calculation of the integral defining $I^{\prime \prime \prime \prime}(0)$ in (3-20) for $\psi$ in $(4-1), \theta \equiv 0$, by finding the "bad integral"

$$
\int_{\mathbb{R}^{n-1}} 6\left(|\bar{\nabla} \psi|^{2}-\psi_{t}^{2}\right) g_{\varepsilon^{2} t} d x .
$$

We begin by computing $g_{\varepsilon \varepsilon}(x, 0)$. To do this we note that the solution $F$ to the Neumann problem for the Laplacian in $D(0)=\left\{(x, t) \in \mathbb{R}^{n}: t>0\right\}$ with boundary function $f$ (see [Stein 1970, Chapter 7]) can be written as

$$
F(x, t)=c_{n} \int_{\mathbb{R}^{n-1}}\left(|x-y|^{2}+t^{2}\right)^{1-n / 2} f(y) d y,
$$

provided $f$ is sufficiently smooth and has rapid enough decay at $\infty$. Here

$$
(n-2) c_{n}=\Gamma\left(\frac{1}{2} n\right) \pi^{-n / 2},
$$

where $\Gamma$ is the Euler Gamma function. We shall often use the fact that for $x, y>0$,

$$
\int_{0}^{\pi / 2} \sin ^{2 x-1} \phi \cos ^{2 y-1} \phi d \phi=\frac{\Gamma(x) \Gamma(y)}{2 \Gamma(x+y)} .
$$

Taking $f=2 \psi \psi_{t}$ we see that

$$
f \in C^{\infty}\left(\mathbb{R}^{n-1}\right) \quad \text { and } \quad|f(x)| \leq c(1+|x|)^{1-2 n} \text { for } x \in \mathbb{R}^{n-1}
$$

and some $c \geq 1$. Using (4-3), (4-5) and Hardy space theory (see [Stein 1970, Chapter 7]) we deduce that

$$
\begin{aligned}
\frac{\partial g_{\varepsilon \varepsilon}}{\partial t}(x, 0) & =-2 c_{n} \lim _{t \rightarrow 0} \frac{\partial^{2}}{\partial t^{2}} \int_{\mathbb{R}^{n-1}}\left(|x-y|^{2}+t^{2}\right)^{1-n / 2} \psi \psi_{t}(y, 0) d y \\
& =2 c_{n} \lim _{t \rightarrow 0} \int_{\mathbb{R}^{n-1}} \bar{\Delta}\left[\left(|x-y|^{2}+t^{2}\right)^{1-n / 2}\right] \psi \psi_{t}(y, 0) d y \\
& =2 c_{n} \int_{\mathbb{R}^{n-1}}|y|^{2-n} \bar{\Delta}\left(\psi \psi_{t}\right)(x-y, 0) d y .
\end{aligned}
$$


We note that

$$
\text { (4-7) } \begin{aligned}
\left(n(n-2)^{2}\right)^{-1} & \bar{\Delta}\left(\psi \psi_{t}\right)(z, 0) \\
= & \frac{\partial^{2}}{\partial z_{1}^{2}}\left(-\frac{n+1}{2 n}\left(1+|z|^{2}\right)^{-n}\right)+\frac{\partial^{2}}{\partial z_{1}^{2}}\left(1+|z|^{2}\right)^{-(n+1)} \\
& -(n+3)\left(1+|z|^{2}\right)^{-(n+1)}+(2 n+2)\left(1+|z|^{2}\right)^{-(n+2)} \\
= & \left(\frac{\partial^{2} f_{1}}{\partial z_{1}^{2}}+\frac{\partial^{2} f_{2}}{\partial z_{1}^{2}}+f_{3}+f_{4}\right)(z) .
\end{aligned}
$$

Using (4-7) in (4-6) we see that at $(x, 0)$,

$$
\left(2 n(n-2)^{2}\right)^{-1} \frac{\partial g_{\varepsilon \varepsilon}}{\partial t}=\frac{\partial^{2}}{\partial x_{1}^{2}}\left(F_{1}+F_{2}\right)+F_{3}+F_{4},
$$

where

$$
F_{i}(x)=c_{n} \int_{\mathbb{R}^{n-1}}|y|^{2-n} \bar{f}_{i}(x-y) d y \quad \text { for } 1 \leq i \leq 4,
$$

and to get $F_{1}, F_{2}$ on the right-hand side of (4-8) we have interchanged the order of differentiation and integration in the corresponding integrals, which is permissible as we see from (4-5). We now introduce spherical coordinates in $\mathbb{R}^{n-1}$. Let $r=|x|$, $\rho=|y|$ and $y_{1}=\rho \cos \theta$. From rotational symmetry we deduce that each $F_{i}$ is radial. Using this and writing $F_{i}(r)$ for $F_{i}(x), 1 \leq i \leq 4$, we see for $n \geq 3$ that each $F_{i}$ has the form

$$
F_{i}(r)=d_{i} / \pi \int_{0}^{\pi} \int_{0}^{\infty} \frac{\sin ^{n-3} \theta}{\left(1+r^{2}-2 r \rho \cos \theta+\rho^{2}\right)^{k_{i}}} d \rho d \theta
$$

where

$$
\begin{array}{ll}
d_{1}=-(n+1) /(2 n), & k_{1}=n, \\
d_{2}=1, & k_{2}=n+1, \\
d_{3}=-(n+3), & k_{3}=n+1, \\
d_{4}=2 n+2, & k_{4}=n+2 .
\end{array}
$$

To evaluate the integrals in (4-10) we shall use contour integration and complex notation. Let $z=x+i y$ and let $\Lambda_{R}$ be the boundary in the complex plane (oriented counterclockwise) of $\{z=x+i y: y>0\} \cap\{z:|z|<R\}$. If

$$
\hat{f}(z, r, \theta)=\left(1+r^{2}-2 r z \cos \theta+z^{2}\right)^{-k}
$$


and $k \geq n$ is a positive integer, then from the residue theorem and simple estimates we have

$$
\begin{aligned}
\pi I(r) & =\int_{0}^{\pi} \int_{0}^{\infty} \frac{\sin ^{n-3} \theta}{\left(1+r^{2}-2 r \rho \cos \theta+\rho^{2}\right)^{k}} d \rho d \theta \\
& =\int_{0}^{\pi / 2} \sin ^{n-3} \theta \lim _{R \rightarrow \infty} \int_{\Lambda_{R}} \hat{f}(z, r, \theta) d z d \theta \\
& =\left.2 \pi i \int_{0}^{\pi / 2} \sin ^{n-3} \theta \operatorname{Res} \hat{f}(\cdot, r, \theta)\right|_{z_{1}} d \theta
\end{aligned}
$$

where $z_{1}$ is the pole of $\hat{f}(\cdot, r, \theta)$ with $\operatorname{Im} z_{1}>0$. To find this residue we note from the quadratic formula that

$$
\hat{f}(z, r, \theta)=\left(z-z_{1}\right)^{-k}\left(z-z_{2}\right)^{-k}
$$

where

$$
z_{1}=r \cos \theta+i \sqrt{1+r^{2} \sin ^{2} \theta} \quad \text { and } \quad z_{2}=\bar{z}_{1} .
$$

Thus

$$
\begin{aligned}
\left.\operatorname{Res} \hat{f}(\cdot, r, \theta)\right|_{z_{1}} & =\frac{1}{(k-1) !} \frac{d^{k-1}}{d z^{k-1}}\left(z-z_{2}\right)^{-k}=(-1)^{k-1} \frac{\Gamma(2 k-1)}{\Gamma(k)^{2}}\left(z_{1}-z_{2}\right)^{1-2 k} \\
& =-i 2^{1-2 k} \frac{\Gamma(2 k-1)}{\Gamma(k)^{2}}\left(1+r^{2} \sin \theta\right)^{1 / 2-k}
\end{aligned}
$$

Using this equality in (4-12) we get

$$
\begin{aligned}
I(r) & =2^{2-2 k} \frac{\Gamma(2 k-1)}{\Gamma(k)^{2}} \int_{0}^{\pi / 2} \frac{\sin ^{n-3} \theta}{\left(1+r^{2} \sin ^{2} \theta\right)^{k-1 / 2}} d \theta \\
& =\left(1+r^{2}\right)^{1 / 2-k} 2^{2-2 k} \frac{\Gamma(2 k-1)}{\Gamma(k)^{2}} \int_{0}^{\pi} \sin ^{n-3} \theta\left(1-\frac{r^{2}}{1+r^{2}} \cos ^{2} \theta\right)^{(1 / 2-k)} d \theta .
\end{aligned}
$$

Next we expand the integrand in (4-13) in a series, using the binomial theorem, and use (4-4) to integrate term by term. If $(a)_{0}=1$ and $(a)_{m}=a(a+1) \ldots(a+m-1)$, for $m$ a positive integer, we find that

$$
\text { (4-14) } \begin{aligned}
\int_{0}^{\pi} \sin ^{n-3} \theta(1 & \left.-\frac{r^{2}}{1+r^{2}} \cos ^{2} \theta\right)^{(1 / 2-k)} d \theta \\
& =\sum_{m=0}^{\infty}\left(\frac{\left(k-\frac{1}{2}\right)_{m}}{m !}\left(\frac{r^{2}}{1+r^{2}}\right)^{m} \int_{0}^{\pi / 2} \sin ^{n-3} \theta \cos ^{2 m} \theta d \theta\right) \\
& =\frac{1}{2} \frac{\Gamma\left(\frac{1}{2}\right) \Gamma\left(\frac{1}{2} n-1\right)}{\Gamma\left(\frac{1}{2}(n-1)\right)} F\left(\frac{1}{2}, k-\frac{1}{2}, \frac{n}{2}-\frac{1}{2}, \frac{r^{2}}{1+r^{2}}\right),
\end{aligned}
$$


where again

$$
F(a, b, c, w)=\sum_{m=0}^{\infty} \frac{(a)_{m}(b)_{m}}{m !(c)_{m}} w^{m}
$$

is the hypergeometric function. Using (4-14) and (4-13) we conclude that

$$
I(r)=A(k)\left(1+r^{2}\right)^{1 / 2-k} F\left(\frac{1}{2}, k-\frac{1}{2}, \frac{n}{2}-\frac{1}{2}, \frac{r^{2}}{1+r^{2}}\right),
$$

where

$$
A(k)=2^{1-2 k} \frac{\Gamma\left(\frac{1}{2}\right) \Gamma\left(\frac{1}{2} n-1\right) \Gamma(2 k-1)}{\Gamma\left(\frac{1}{2} n-\frac{1}{2}\right) \Gamma(k)^{2}} .
$$

Next, from (4-15), (4-11), (4-10), we deduce that

$$
\begin{aligned}
& F_{1}(r)=-\frac{n+1}{2 n} A(n)\left(1+r^{2}\right)^{1 / 2-n} F\left(\frac{1}{2}, n-\frac{1}{2}, \frac{n}{2}-\frac{1}{2}, \frac{r^{2}}{1+r^{2}}\right), \\
& F_{2}(r)=A(n+1)\left(1+r^{2}\right)^{-n-1 / 2} F\left(\frac{1}{2}, n+\frac{1}{2}, \frac{n}{2}-\frac{1}{2}, \frac{r^{2}}{1+r^{2}}\right), \\
& F_{3}(r)=-(n+3) A(n+1)\left(1+r^{2}\right)^{-n-1 / 2} F\left(\frac{1}{2}, n+\frac{1}{2}, \frac{n}{2}-\frac{1}{2}, \frac{r^{2}}{1+r^{2}}\right), \\
& F_{4}(r)=(2 n+2) A(n+2)\left(1+r^{2}\right)^{-n-3 / 2} F\left(\frac{1}{2}, n+\frac{3}{2}, \frac{n}{2}-\frac{1}{2}, \frac{r^{2}}{1+r^{2}}\right) .
\end{aligned}
$$

To compute derivatives of $I(r)$ we note that if $z=r^{2} /\left(1+r^{2}\right)$, then $I(r)$ can be written as a constant multiple of $(1-z)^{b} F(a, b, c, z)$ for suitably chosen $a, b, c$. Then $\partial z / \partial x_{1}=2 x_{1}(1-z)^{2}$ and

$$
\begin{aligned}
\frac{\partial}{\partial x_{1}}\left((1-z)^{b} F(a, b, c, z)\right) & =2 x_{1}(1-z)^{b+1}\left(-b F(a, b, z)+(1-z) \frac{\partial F}{\partial z}(a, b, c, z)\right) \\
& =2 x_{1}(1-z)^{b+1}(a-c)(b / c), F(a, b+1, c+1) .
\end{aligned}
$$

Differentiating this equality once again we find with $x_{1}=r \cos \theta$ that

$$
\begin{aligned}
\frac{\partial^{2}}{\partial x_{1}^{2}} I(r)=B(k)(1+ & \left.r^{2}\right)^{-k-1 / 2} F\left(\frac{1}{2}, k+\frac{1}{2}, \frac{n}{2}+\frac{1}{2}, \frac{r^{2}}{1+r^{2}}\right) \\
& +C(k) \cos ^{2} \theta r^{2}\left(1+r^{2}\right)^{-k-3 / 2} F\left(\frac{1}{2}, k+\frac{3}{2}, \frac{n}{2}+\frac{3}{2}, \frac{r^{2}}{1+r^{2}}\right),
\end{aligned}
$$

where

$$
\begin{aligned}
& B(k)=-(2 k-1) 2^{1-2 k} \frac{\Gamma\left(\frac{1}{2}\right) \Gamma\left(\frac{n}{2}\right) \Gamma(2 k-1)}{\Gamma\left(\frac{n}{2}+\frac{1}{2}\right) \Gamma(k)^{2}}, \\
& C(k)=\left(4 k^{2}-1\right) 2^{1-2 k} \frac{\Gamma\left(\frac{1}{2}\right) \Gamma\left(\frac{n}{2}+1\right) \Gamma(2 k-1)}{\Gamma\left(\frac{n}{2}+\frac{3}{2}\right) \Gamma(k)^{2}} .
\end{aligned}
$$


From these equalities we see that

$(4-17)$

$$
\begin{aligned}
\frac{\partial^{2}}{\partial z_{1}^{2}} F_{1}(r)= & \frac{(n+1)}{2 n} B(n)\left(1+r^{2}\right)^{-n-1 / 2} F\left(\frac{1}{2}, n+\frac{1}{2}, n / 2+\frac{1}{2}, \frac{r^{2}}{1+r^{2}}\right) \\
& +\frac{(n+1)}{2 n} C(n) \cos ^{2} \theta r^{2}\left(1+r^{2}\right)^{-n-3 / 2} F\left(\frac{1}{2}, n+\frac{3}{2}, \frac{n}{2}+\frac{3}{2}, \frac{r^{2}}{1+r^{2}}\right) \\
= & G_{11}(r)+G_{12}(r) \cos ^{2} \theta,
\end{aligned}
$$

$$
\begin{aligned}
\frac{\partial^{2}}{\partial z_{1}^{2}} F_{2}(r)= & B(n+1)\left(1+r^{2}\right)^{-n-3 / 2} F\left(\frac{1}{2}, n+\frac{3}{2}, \frac{n}{2}+\frac{1}{2}, \frac{r^{2}}{1+r^{2}}\right) \\
& +C(n+1) \cos ^{2} \theta r^{2}\left(1+r^{2}\right)^{-n-5 / 2} F\left(\frac{1}{2}, n+\frac{5}{2}, \frac{n}{2}+\frac{3}{2}, \frac{r^{2}}{1+r^{2}}\right) \\
= & G_{21}(r)+G_{22}(r) \cos ^{2} \theta .
\end{aligned}
$$

Combining these two equalities with (4-16), we see that $\partial g_{\varepsilon \varepsilon} / \partial t$ is calculated in terms of the hypergeometric function.

Next we write $\left(|\bar{\nabla} \psi|^{2}-\psi_{t}^{2}\right)(x, 0)$ in polar coordinates. If $r=|x|$ and $x_{1}=$ $r \cos \theta$, we have

$$
\begin{aligned}
|\bar{\nabla} \psi|^{2}-\psi_{t}^{2}(x, 0) & =-\frac{2 n^{2}(n-2)^{2} r^{2} \cos ^{2} \theta}{\left(r^{2}+1\right)^{n+2}}+\frac{n(n-2)^{3} r^{2} \cos ^{2} \theta}{\left(r^{2}+1\right)^{n+1}}+\frac{(n-2)^{2}}{\left(r^{2}+1\right)^{n}} \\
& =g_{1}(r) \cos ^{2} \theta+g_{2}(r) \cos ^{2} \theta+g_{3}(r) .
\end{aligned}
$$

To find the integrals with integrand involving $g_{i}(\partial / \partial t) g_{\varepsilon \varepsilon}$, for $1 \leq i \leq 3$, we first consider integrals of the form

$$
J(b, h, k, l)=\int_{0}^{\infty} r^{l}\left(1+r^{2}\right)^{-h-k} F\left(\frac{1}{2}, k, b, \frac{r^{2}}{1+r^{2}}\right) d r,
$$

when $l$ equals $n-2, n$, or $n+2$, and $h, k>0$ with $h+k \geq 2 n+\frac{1}{2}$. Interchanging the integration and summation signs in this integral (which is permissible by the Tonelli theorem) and using the substitution $r=\tan \phi$, we get

$$
\begin{aligned}
J(b, h, k, l) & =\sum_{m=0}^{\infty} \frac{\left(\frac{1}{2}\right)_{m}(k)_{m}}{(b)_{m} m !} \int_{0}^{\infty} \frac{r^{2 m+l}}{\left(1+r^{2}\right)^{m+h+k}} d r \\
& =\sum_{m=0}^{\infty} \frac{\left(\frac{1}{2}\right)_{m}(k)_{m}}{m !(b)_{m}} \int_{0}^{\pi / 2}(\cos \phi)^{2 h+2 k-2-l}(\sin \phi)^{2 m+l} d \phi \\
& =\frac{\Gamma\left(h+k-\frac{l}{2}-\frac{1}{2}\right) \Gamma\left(\frac{l}{2}+\frac{1}{2}\right)}{2 \Gamma(h+k)} \sum_{m=0}^{\infty} \frac{\left(\frac{l}{2}+\frac{1}{2}\right)_{m}\left(\frac{1}{2}\right)_{m}(k)_{m}}{(h+k)_{m}(b)_{m} m !} .
\end{aligned}
$$


We first use (4-18) with $l=n-2$ and $b=\frac{n}{2}-\frac{1}{2}$ to get

$$
\begin{array}{r}
J\left(\frac{n}{2}-\frac{1}{2}, h, k, n-2\right)=\frac{\Gamma\left(h+k-\frac{n}{2}+\frac{1}{2}\right) \Gamma\left(\frac{n}{2}-\frac{1}{2}\right)}{2 \Gamma(h+k)} \sum_{m=0}^{\infty} \frac{\left(\frac{1}{2}\right)_{m}(k)_{m}}{(k+h)_{m} m !} \\
\times \frac{\Gamma\left(h+k-\frac{n}{2}+\frac{1}{2}\right) \Gamma\left(\frac{n}{2}-\frac{1}{2}\right)}{2 \Gamma(h+k)) F\left(\frac{1}{2}, k, h+k, 1\right)} \\
=\frac{\Gamma\left(\frac{n}{2}-\frac{1}{2}\right) \Gamma\left(h+k-\frac{n}{2}+\frac{1}{2}\right) \Gamma\left(h-\frac{1}{2}\right)}{2 \Gamma(h) \Gamma\left(h+k-\frac{1}{2}\right)} .
\end{array}
$$

Here we have used the fact that

(4-21) $\quad F(\alpha, \beta, \gamma, 1)=\frac{\Gamma(\gamma-\alpha-\beta) \Gamma(\gamma)}{\Gamma(\gamma-\alpha) \Gamma(\gamma-\beta)} \quad$ if $\alpha, \beta, \gamma>0$ and $\gamma-\alpha-\beta>0$.

From (4-20) with $h=n$ and $k=n+\frac{1}{2},(4-16)$ for $F_{3}$, and (4-4) we get

$$
\begin{aligned}
H_{1} & =\int_{0}^{\infty} \int_{0}^{\pi} \sin ^{n-3} \theta F_{3}(r) g_{3}(r) r^{n-2} d r \\
& =-(n-2)^{2}(n+3) A(n+1) \frac{\Gamma\left(\frac{n}{2}-1\right) \Gamma\left(\frac{1}{2}\right)}{\Gamma\left(\frac{n}{2}-\frac{1}{2}\right)} J\left(\frac{n}{2}-\frac{1}{2}, n, n+\frac{1}{2}, n-2\right) \\
& =-8(n+3)(n-2)^{2} n^{2} D,
\end{aligned}
$$

where

$$
D=\frac{\pi \Gamma\left(\frac{n}{2}-1\right)^{2} \Gamma\left(\frac{3 n}{2}+1\right) \Gamma\left(n-\frac{1}{2}\right)}{2^{4+2 n} \Gamma\left(\frac{n}{2}-\frac{1}{2}\right) \Gamma(n+1)^{3}} .
$$

Likewise from (4-20) with $h=n$ and $k=n+\frac{3}{2}$, (4-16) for $F_{4}$, and (4-4) we get

$$
\begin{aligned}
H_{2} & =\int_{0}^{\infty} \int_{0}^{\pi} \sin ^{n-3} \theta F_{4}(r) g_{3}(r) r^{n-2} d \theta d r \\
& =2(3 n+2)(2 n+1) n(n-2)^{2} D .
\end{aligned}
$$

Next we put $b=\frac{n}{2}-\frac{1}{2}$ and $l=n$ in the sum on the last line of (4-19). We deduce $\sum_{m=0}^{\infty} \frac{\left(\frac{n}{2}+\frac{1}{2}\right)_{m}\left(\frac{1}{2}\right)_{m}(k)_{m}}{(h+k)_{m}\left(\frac{n}{2}-\frac{1}{2}\right)_{m} m !}=F\left(\frac{1}{2}, k, h+k\right)+\frac{k}{(h+k)(n-1)} F\left(\frac{3}{2}, k+1, h+k+1\right)$.

Using this together with (4-21) and (4-19) we find after simplifying that (4-25) $J\left(\frac{n}{2}-\frac{1}{2}, h, k, n\right)$

$$
=\frac{\Gamma\left(h+k-\frac{n}{2}-\frac{1}{2}\right) \Gamma\left(\frac{n}{2}+\frac{1}{2}\right) \Gamma\left(h-\frac{3}{2}\right)}{4(n-1) \Gamma\left(h+k-\frac{1}{2}\right) \Gamma(h)}((n-1)(2 h-3)+2 k) .
$$


Using (4-25) with $b=\frac{n}{2}-\frac{1}{2}, h=n+2, k=n+\frac{1}{2}$ and $l=n$, together with (4-16) for $F_{3}$ and (4-4), we get

$$
\begin{aligned}
H_{3} & =\int_{0}^{\infty} \int_{0}^{\pi / 2} \sin ^{n-3} \theta \cos ^{2} \theta F_{3}(r) g_{1}(r) r^{n-2} d r \\
& =2 n^{2}(n-2)^{2}(n+3) A(n+1) \frac{\Gamma\left(\frac{n}{2}-1\right) \Gamma\left(\frac{1}{2}\right)}{(n-1) \Gamma\left(\frac{n}{2}-\frac{1}{2}\right)} J\left(\frac{n}{2}-\frac{1}{2}, n+2, n+\frac{1}{2}, n\right) \\
& =\frac{(2 n-1)(3 n+2)(n-2)^{2} n^{3}(n+3)}{2\left(n^{2}-1\right)} D .
\end{aligned}
$$

Arguing as above with $b=\frac{n}{2}-\frac{1}{2}, h=n+2, k=n+\frac{3}{2}, l=n$, we also obtain

$$
\begin{aligned}
H_{4}=\int_{0}^{\infty} \int_{0}^{\pi / 2} & \sin ^{n-3} \theta \cos ^{2} \theta F_{4}(r) g_{1}(r) r^{n-2} d r \\
& =-\frac{n^{2}(n-2)^{2}(2 n-1)\left(2 n^{2}+n+2\right)(3 n+2)(3 n+4)}{8(n+1)^{2}(n-1)} D .
\end{aligned}
$$

Next with $b, l$ as above, $h=n+1$, and $k=n+\frac{1}{2}$, we find

$$
\begin{aligned}
H_{5}=\int_{0}^{\infty} \int_{0}^{\pi / 2} \sin ^{n-3} \theta \cos ^{2} \theta F_{3}(r) & g_{2}(r) r^{n} d r \\
& =-\frac{\left(2 n^{2}-n+2\right) n(n-2)^{3}(n+3)}{n-1} D .
\end{aligned}
$$

Also for $b, l$ as above and $h=n+1, k=n+\frac{3}{2}$,

$$
\begin{aligned}
H_{6}=\int_{0}^{\infty} \int_{0}^{\pi / 2} \sin ^{n-3} \theta \cos ^{2} \theta F_{4}(r) & g_{2}(r) r^{n-2} d r \\
& =\frac{n(n-2)^{3}(3 n+2)\left(2 n^{2}-n+4\right)}{2(n-1)} D .
\end{aligned}
$$

To continue, we note that if $b=\frac{n}{2}+\frac{1}{2}$ and $l=n$ in the sum in (4-19), one deduces as in (4-20) that

$$
J\left(\frac{n}{2}+\frac{1}{2}, h, k, n\right)=\frac{\Gamma\left(h+k-\frac{n}{2}-\frac{1}{2}\right) \Gamma\left(\frac{n}{2}+\frac{1}{2}\right) \Gamma\left(h-\frac{1}{2}\right)}{2 \Gamma(h) \Gamma\left(h+k-\frac{1}{2}\right)} .
$$

In view of (4-30) with $b=\frac{n}{2}+\frac{1}{2}, h=n+2, k=n+\frac{1}{2}, l=n$, we find from (4-18) and the first equality in (4-17) that

$$
\text { (4-31) } \begin{aligned}
H_{7}=\int_{0}^{\infty} \int_{0}^{\pi / 2} \sin ^{n-3} \theta \cos ^{2} \theta G_{11}(r) & g_{1}(r) r^{n-2} d r \\
& =-\frac{(3 n+2)(2 n-1)(n-2)^{3} n^{2}}{2(n-1)} D .
\end{aligned}
$$


With $b, h, l$ as in (4-31) and $k=n+\frac{3}{2}$ we deduce as above that

(4-32) $\quad H_{8}=\int_{0}^{\infty} \int_{0}^{\pi / 2} \sin ^{n-3} \theta \cos ^{2} \theta G_{21}(r) g_{1}(r) r^{n-2} d r$

$$
=\frac{n^{2}(n-2)^{3}(3 n+2)(3 n+4)\left(4 n^{2}-1\right)}{8(n-1)(n+1)^{2}} D .
$$

Next we repeat our program for the integrals in (4-31), (4-32) with $h=n+1$ and all other parameters as in (4-31), (4-32) respectively. We get

$$
\begin{aligned}
H_{9}=\int_{0}^{\infty} \int_{0}^{\pi / 2} \sin ^{n-3} \theta \cos ^{2} \theta G_{11}(r) g_{2}(r) r^{n-2} d r \\
=\frac{(n-2)^{4}(n+1)(2 n-1) n}{n-1} D,
\end{aligned}
$$

(4-34) $\quad H_{10}=\int_{0}^{\infty} \int_{0}^{\pi / 2} \sin ^{n-3} \theta \cos ^{2} \theta G_{21}(r) g_{2}(r) r^{n-1} d r$

$$
=-\frac{n(n-2)^{4}(3 n+2)(2 n-1)}{2(n-1)} D .
$$

As in (4-30) we see that if $l=n+2$ and $b=\frac{n}{2}+\frac{3}{2}$, then

$$
J\left(\frac{n}{2}+\frac{3}{2}, h, k, n+2\right)=\frac{\Gamma\left(h+k-\frac{n}{2}-\frac{3}{2}\right) \Gamma\left(\frac{n}{2}+\frac{3}{2}\right) \Gamma\left(h-\frac{1}{2}\right)}{2 \Gamma(h) \Gamma\left(h+k-\frac{1}{2}\right)} .
$$

Using (4-35) with $b, l$ as in (4-30) and $h=n+2, k=n+\frac{3}{2}$ we find

$$
\begin{aligned}
H_{11}=\int_{0}^{\infty} \int_{0}^{\pi / 2} \sin ^{n-3} \theta \cos ^{4} \theta G_{12}(r) & g_{1}(r) r^{n-2} d r \\
& =\frac{3(3 n+2)(n-2)^{3} n^{3}\left(4 n^{2}-1\right)}{8(n+1)^{2}(n-1)} D .
\end{aligned}
$$

Also for $b, l$, as in (4-35) we have with $h=n+2, k=n+5 / 2$,

$$
\begin{aligned}
H_{12}=\int_{0}^{\infty} \int_{0}^{\pi / 2} \sin ^{n-3} \theta \cos ^{4} \theta G_{22}(r) g_{1}(r) r^{n-2} d r \\
=-\frac{3(3 n+2)(3 n+4)(n-2)^{3} n^{3}\left(4 n^{2}-1\right)}{16\left(n^{2}-1\right)(n+1)^{2}} D .
\end{aligned}
$$

Next we repeat the argument that gave us (4-34), (4-35) with $b, l, k$ as in these displays and $h=n+1$. Doing this we get

(4-38) $H_{13}=\int_{0}^{\infty} \int_{0}^{\pi / 2} \sin ^{n-3} \theta \cos ^{4} \theta G_{12}(r) g_{2}(r) r^{n-2} d r$

$$
=-\frac{3(n-2)^{4} n^{2}(2 n-1)}{2(n-1)} D,
$$




$$
\begin{aligned}
H_{14}=\int_{0}^{\infty} \int_{0}^{\pi / 2} \sin ^{n-3} \theta \cos ^{4} \theta G_{22}(r) g_{2}(r) r^{n-2} d r \\
=\frac{3(2 n+3)(3 n+2)(n-2)^{4} n^{2}(2 n-1)}{8\left(n^{2}-1\right)(n+1)} D .
\end{aligned}
$$

Next we note from integrating by parts and radial symmetry that

with

$$
\begin{aligned}
\int_{0}^{\infty} \int_{0}^{\pi / 2} \sin ^{n-3} \theta g_{3}(r) \frac{\partial^{2}}{\partial x_{1}^{2}}\left(F_{1}+F_{2}\right)(r) r^{n-2} d \theta d r \\
=\int_{0}^{\infty} \int_{0}^{\pi / 2} \sin ^{n-3} \theta \frac{\partial^{2} g_{3}}{\partial x_{1}^{2}}(r)\left(F_{1}+F_{2}\right)(r) r^{n-2} d \theta d r \\
=H_{15}+H_{16},
\end{aligned}
$$

$$
\begin{aligned}
& H_{15}=-2 n(n-2)^{2} \int_{0}^{\infty} \int_{0}^{\pi / 2} \sin ^{n-3} \theta\left(F_{1}+F_{2}\right)(r) \frac{r^{n-2}}{\left(r^{2}+1\right)^{n+1}} d \theta, \\
& H_{16}=4 n(n+1)(n-2)^{2} \int_{0}^{\infty} \int_{0}^{\pi / 2} \sin ^{n-3} \theta \cos ^{2} \theta\left(F_{1}+F_{2}\right)(r) \frac{r^{n}}{\left(r^{2}+1\right)^{n+2}} d \theta .
\end{aligned}
$$

To find $H_{15}$ we first write this integral as a sum of two integrals involving $F_{1}, F_{2}$. We then use $b=\frac{n}{2}-\frac{1}{2}, h=n+1, k=n-\frac{1}{2}, l=n-2$ in (4-20) to evaluate the integral involving $F_{1}$ and then $b=\frac{n}{2}-\frac{1}{2}, h=n+1, k=n+\frac{1}{2}, l=n-2$ in (4-20) to evaluate the integral involving $F_{2}$. Altogether we get

$$
H_{15}=\left(8 n^{2}(n-2)^{2}(n+1)-2 n(n-2)^{2}(2 n-1)(3 n+2)\right) D .
$$

Next we use (4-25) and argue as above to find $(4-42)$

$$
H_{16}=\left(-\frac{2(n+1)(n-2)^{2} n\left(2 n^{2}+n-2\right)}{n-1}+\frac{(3 n+2)(n-2)^{2} n^{2}(2 n-1)}{n-1}\right) D .
$$

Combining the integrals in (4-22)-(4-42) and simplifying we find from (4-8) that

$$
\begin{array}{rl}
\int_{\mathbb{R}^{n-1}} & 6 \frac{\partial g_{\varepsilon \varepsilon}}{\partial t}\left(|\bar{\nabla} \psi|^{2}-\psi_{t}^{2}\right) d x \\
& =\alpha_{n} \int_{0}^{\pi / 2} \int_{0}^{\infty} \sin ^{n-3} \theta \frac{\partial g_{\varepsilon \varepsilon}}{\partial t}(r, \theta) r^{n-2} d r d \theta=12 n(n-2)^{2} \sum_{i=1}^{16} H_{i} \\
& =-\alpha_{n} \frac{3 n^{3}(n-2)^{4}\left(16+16 n-72 n^{2}-44 n^{3}-11 n^{4}+12 n^{6}\right)}{4(n-1)(n+1)^{3}} D
\end{array}
$$

where

$$
\alpha_{n}=\frac{2 \pi^{n / 2-1}}{\Gamma\left(\frac{n}{2}-1\right)} .
$$




\section{Proof of Theorem 3}

We first calculate the integral involving the rest of the integrand in $I^{\prime \prime \prime \prime}(0)$ and then use Stirling's formula to finally get Theorem 3. To begin we note as before that if $x \in \mathbb{R}^{n-1}$ and $r=|x|$, then

$$
|\bar{\nabla} \psi|^{2}(x, 0)=\frac{(n-2)^{2}}{\left(r^{2}+1\right)^{n}}-\frac{(n-2)^{2} n^{2} x_{1}^{2}}{\left(r^{2}+1\right)^{n+2}}+\frac{(n-2)^{3} n x_{1}^{2}}{\left(r^{2}+1\right)^{n+1}}
$$

and

$$
\begin{aligned}
|\bar{\nabla} \psi|^{4}(x, 0)=- & \frac{2(n-2)^{4} n^{2} x_{1}^{2}}{\left(r^{2}+1\right)^{2 n+2}}+\frac{2(n-2)^{5} n x_{1}^{2}}{\left(r^{2}+1\right)^{2 n+1}}-\frac{2(n-2)^{5} n^{3} x_{1}^{4}}{\left(r^{2}+1\right)^{2 n+3}} \\
& +\frac{(n-2)^{4}}{\left(r^{2}+1\right)^{2 n}}+\frac{(n-2)^{4} n^{4} x_{1}^{4}}{\left(r^{2}+1\right)^{2 n+4}}+\frac{(n-2)^{6} n^{2} x_{1}^{4}}{\left(r^{2}+1\right)^{2 n+2}} .
\end{aligned}
$$

Introducing spherical coordinates $x_{1}=r \cos \theta$ in (5-2) and integrating the resulting expression term by term, using once again the substitution $r=\tan \phi$ as well as (4-4), we obtain

$$
\int_{\mathbb{R}^{n-1}} 6|\bar{\nabla} \psi|^{4} d x=\alpha_{n} \frac{3(n-2)^{3}\left(3 n^{4}+8 n^{3}+133 n^{2}+392 n+336\right)}{16(n+1)(2 n+3)(2 n+1)} E,
$$

where $\alpha_{n}$ is defined after (4-43) and

$$
E=\frac{\sqrt{\pi} \Gamma(1+n / 2) \Gamma\left(\frac{3 n}{2}+\frac{1}{2}\right)}{\Gamma(2 n)}=\frac{\pi \Gamma(1+n / 2) \Gamma\left(\frac{3 n}{2}+\frac{1}{2}\right)}{\Gamma(n) \Gamma\left(n+\frac{1}{2}\right) 2^{2 n-1}},
$$

by Legendre's duplication formula for the $\Gamma$ function. We note that

$$
\psi_{t}^{2}(x, 0)=\frac{(n-2)^{2} n^{2} x^{2}}{\left(r^{2}+1\right)^{n+2}} .
$$

Multiplying (5-5) by (5-1) and integrating we find that

$$
\int_{\mathbb{R}^{n-1}} 12 \psi_{t}^{2}|\bar{\nabla} \psi|^{2} d x=\alpha_{n} \frac{3(n-2)^{3}\left(3 n^{3}+n^{2}+4 n+24\right)(3 n+1)}{8(2 n+1)(n+1)(2 n+3)} E .
$$

Also squaring (5-4) and integrating we find

$$
\int_{\mathbb{R}^{n-1}} 2 \psi_{t}^{4} d x=\alpha_{n} \frac{9(n-2)^{3} n^{2}(3 n+1)}{16(2 n+1)(2 n+3)} E .
$$

Next we note that

$$
\bar{\Delta} \psi(x, 0)=-\frac{n(n-2) x}{\left(r^{2}+1\right)^{n / 2+1}}+\frac{n\left(n^{2}-4\right) x}{\left(r^{2}+1\right)^{n / 2+2}} .
$$

Multiplying together (5-8), (4-1), (5-1) and integrating we get

$$
\text { (5-9) } \int_{\mathbb{R}^{n-1}} 12 \psi|\bar{\nabla} \psi|^{2} \bar{\Delta} \psi d x=-\alpha_{n} \frac{9(n-2)^{3}\left(3 n^{4}+4 n^{3}+n^{2}+32 n+48\right)}{8(2 n+1)(2 n+3)(n+1)} E \text {. }
$$


To handle the last term in the integral observe that

$$
\text { (5-10) } \begin{aligned}
\frac{\partial|\bar{\nabla} \psi|^{2}}{\partial t} & (x, 0) \\
& =-\frac{2 n(n-2)^{2}}{\left(r^{2}+1\right)^{n+1}}+\frac{2 n^{2}(n-2)^{2}(n+2) x^{2}}{\left(r^{2}+1\right)^{n+3}}-\frac{2 n(n-2)^{2}\left(n^{2}-2\right) x^{2}}{\left(r^{2}+1\right)^{n+2}} .
\end{aligned}
$$

Multiplying together (5-10), the positive square root of (5-5), (4-1) and integrating using (4-2) we obtain

$$
\int_{\mathbb{R}^{n-1}} 24 \psi \psi_{t}\left\langle\bar{\nabla} \psi, \bar{\nabla} \psi_{t}\right\rangle d x=\alpha_{n} \frac{3(n-2)^{3}\left(3 n^{3}+7 n^{2}-2 n-12\right)(3 n+1)}{4(n+1)(2 n+1)(2 n+3)} E .
$$

Adding together (5-3), (5-6), (5-7), (5-9), and (5-11) we conclude that

$$
\begin{aligned}
\int_{\mathbb{R}^{n-1}}\left(12 \psi|\bar{\nabla} \psi|^{2} \bar{\Delta} \psi+24 \psi \psi_{t}\left\langle\bar{\nabla} \psi, \bar{\nabla} \psi_{t}\right\rangle\right. & \left.+2 \psi_{t}^{4}+12|\bar{\nabla} \psi|^{2} \psi_{t}^{2}+6|\bar{\nabla} \psi|^{4}\right) d x \\
& =\alpha_{n} \frac{3\left(3 n^{3}+2 n^{2}+7 n+2\right)(n-2)^{3}}{2(n+1)(2 n+1)} E .
\end{aligned}
$$

Combining this with (4-43) we get

$$
\begin{array}{r}
I^{\prime \prime \prime \prime}(0)=-\alpha_{n} \frac{3 n^{3}(n-2)^{4}\left(16+16 n-72 n^{2}-44 n^{3}-11 n^{4}+12 n^{6}\right)}{4(n-1)(n+1)^{3}} D \\
+\alpha_{n} \frac{3\left(3 n^{3}+2 n^{2}+7 n+2\right)(n-2)^{3}}{2(n+1)(2 n+1)} \\
=A\left(-\frac{1}{16} L \frac{n\left(16+16 n-72 n^{2}-44 n^{3}-11 n^{4}+12 n^{6}\right)}{(n+1)^{2}(n-1)}\right. \\
\left.+\frac{3 n^{3}+2 n^{2}+7 n+2}{2 n+1}\right),
\end{array}
$$

where

$$
\begin{aligned}
& A=\frac{3 n \alpha_{n} \pi(n-2)^{4} \Gamma\left(\frac{n}{2}-1\right) \Gamma\left(\frac{3 n}{2}+\frac{1}{2}\right) 2^{-2 n-2}}{\Gamma(n) \Gamma\left(n+\frac{1}{2}\right)(n+1)}, \\
& L=\frac{\Gamma\left(n+\frac{1}{2}\right) \Gamma\left(n-\frac{1}{2}\right) \Gamma\left(\frac{n}{2}-1\right) \Gamma\left(\frac{3 n}{2}+1\right)}{\Gamma(n+1) \Gamma(n+1) \Gamma\left(\frac{n}{2}-\frac{1}{2}\right) \Gamma\left(\frac{3 n}{2}+\frac{1}{2}\right)} .
\end{aligned}
$$

In order to show that (5-13) is positive we need to estimate $L$ from above. Substituting $\Gamma\left(\frac{3 n}{2}+1\right)=\frac{3 n}{2} \Gamma\left(\frac{3 n}{2}\right)$ and $\Gamma(n+1)=n \Gamma(n)$ once gives

$$
L=\frac{3}{2} \frac{\Gamma\left(n+\frac{1}{2}\right) \Gamma\left(n-\frac{1}{2}\right) \Gamma\left(\frac{n}{2}-1\right) \Gamma\left(\frac{3 n}{2}\right)}{\Gamma(n+1) \Gamma(n) \Gamma\left(\frac{n}{2}-\frac{1}{2}\right) \Gamma\left(\frac{3 n}{2}+\frac{1}{2}\right)} .
$$


This gives four ratios of Gamma functions, in the order they appear, of the form $\Gamma(z) / \Gamma\left(z+\frac{1}{2}\right)$. Using Stirling's formula (see [Ahlfors 1978, p. 203]) we see that

$$
\frac{\Gamma(z)}{\Gamma(z+1 / 2)}=\left(\frac{z}{z+\frac{1}{2}}\right)^{z} \frac{e^{1 / 2}}{z^{1 / 2}} e^{J(z)-J(z+1 / 2)},
$$

where

$$
J(z)=\frac{1}{\pi} \int_{0}^{\infty} \frac{z}{\eta^{2}+z^{2}} \log \frac{1}{1-e^{-2 \pi \eta}} d \eta \quad \text { for } z=n+\frac{1}{2}, n-\frac{1}{2}, \frac{n}{2}-1, \frac{3 n}{2}+1 .
$$

Next observe that

$$
\left(\frac{z}{z+\frac{1}{2}}\right)^{z}=\exp \left(z \ln \left(1-\frac{1}{2 z+1}\right)\right) \leq \exp \left(z \frac{-1}{2 z+1}\right) \leq \exp \left(-\frac{n-2}{2(n-1)}\right)
$$

for all $z$ occurring above. Therefore,

$$
L \leq \frac{3}{2} \frac{e^{-2(n-2) /(n-1)} e^{2}}{\sqrt{\left(n+\frac{1}{2}\right)\left(n-\frac{1}{2}\right)\left(\frac{n}{2}-1\right)\left(\frac{3 n}{2}+1\right)}} S \leq \frac{\sqrt{3} e^{2 /(n-2)}}{n^{2} \sqrt{\left(1-\frac{1}{2 n}\right)\left(1-\frac{2}{n}\right)}} S
$$

for $n \geq 3$, where $S$ stands for $\exp \left(J\left(n+\frac{1}{2}\right)-J(n+1)+J\left(n-\frac{1}{2}\right)-J(n)+J\left(\frac{n}{2}-1\right)-J\left(\frac{n}{2}-\frac{1}{2}\right)+J\left(\frac{3 n}{2}\right)-J\left(\frac{3 n}{2}+\frac{1}{2}\right)\right)$.

We estimate $J(z)-J\left(z+\frac{1}{2}\right)$ using the formula above and the mean value theorem $\left(z \leq \xi \leq z+\frac{1}{2}\right)$

$$
\begin{aligned}
\left|J(z)-J\left(z+\frac{1}{2}\right)\right| & =\frac{1}{2 \pi}\left|\int_{0}^{\infty} \frac{\eta^{2}-\xi^{2}}{\left(\eta^{2}+\xi^{2}\right)^{2}} \log \left(\frac{1}{1-e^{-2 \pi \eta}}\right) d \eta\right| \\
& \leq \frac{1}{2 \pi \xi^{2}} \int_{0}^{\infty} \log \frac{1}{1-e^{-2 \pi \eta}} d \eta \leq \frac{1}{24 \xi^{2}} \leq \frac{1}{24 z^{2}},
\end{aligned}
$$

where we have also used the fact that the last integral is $\pi / 12$ (see [Ahlfors 1978, p. 205]). Again using that the least $z$ above is $z=\frac{1}{2}(n-2)$ we see that

$$
S \leq\left(\exp \frac{1}{24\left(\frac{n}{2}-1\right)^{2}}\right)^{4}=\exp \frac{2}{3(n-2)^{2}} .
$$

Using this inequality in (5-14) we have

$$
L \leq \frac{\sqrt{3} \exp \left(\frac{2}{n-2}+\frac{2}{3(n-2)^{2}}\right)}{n^{2} \sqrt{\left(1-\frac{1}{2 n}\right)\left(1-\frac{2}{n}\right)}} .
$$

We also have for $n \geq 3$

$$
\frac{n\left(16+16 n-72 n^{2}-44 n^{3}-11 n^{4}+12 n^{6}\right)}{(n+1)^{2}(n-1)} \leq \frac{12 n^{7}}{n^{3}}=12 n^{4} .
$$


Combining (5-16) and (5-15) we deduce that

$$
\begin{aligned}
& \frac{1}{16} \frac{\Gamma\left(n+\frac{1}{2}\right) \Gamma\left(n-\frac{1}{2}\right) \Gamma\left(\frac{n}{2}-1\right) \Gamma\left(\frac{3 n}{2}+1\right)}{\Gamma(n+1)^{2} \Gamma\left(\frac{n}{2}-\frac{1}{2}\right) \Gamma\left(\frac{3 n}{2}+\frac{1}{2}\right)} \\
& \times \frac{n\left(16+16 n-72 n^{2}-44 n^{3}-11 n^{4}+12 n^{6}\right)}{(n+1)^{2}(n-1)} \\
& \leq \frac{3 n^{2} \sqrt{3} \exp \left(\frac{2}{n-2}+\frac{1}{6(n-2)^{2}}\right)}{4 \sqrt{\left(1-\frac{1}{2 n}\right)\left(1-\frac{2}{n}\right)}} \leq \frac{3 n^{3}+2 n^{2}+7 n+2}{2 n+1}
\end{aligned}
$$

for $n \geq 100$, as is easily seen. From (5-17) and (5-13) we conclude that $I^{\prime \prime \prime \prime}(0) \geq 0$ for $n \geq 100$. For $3 \leq n \leq 100$ one can check (5-13) directly to see that $I^{\prime \prime \prime \prime}(0) \geq 0$. This is easily done using a computer. In fact it turns out that (5-17) is valid for $n \geq 23$ so that one need only verify directly that (5-13) is positive for $3 \leq n \leq 23$. The proof of Theorem 3 is now complete.

\section{Proof of Theorem 4}

Let $\psi$ be as in Theorem 3 and put $\theta=\psi \psi_{t}$. From (3-5) we have $g_{\varepsilon^{2}} \equiv 0$ so $g_{\varepsilon^{2} t} \equiv 0$ and the "bad term" in the last calculation now vanishes. Also note that

(6-1) $\int_{\mathbb{R}^{n-1}}\left\langle\bar{\nabla} \psi, \bar{\nabla}\left(\psi \psi_{t}\right)\right\rangle \psi_{t} d x=\int_{\mathbb{R}^{n-1}}|\bar{\nabla} \psi|^{2} \psi_{t}^{2} d x+\int_{\mathbb{R}^{n-1}}\left\langle\bar{\nabla} \psi_{t}, \bar{\nabla} \psi\right\rangle \psi \psi_{t} d x$.

Multiplying by -24 , substituting into (3-20) and collecting terms we see that

$$
I^{\prime \prime \prime \prime}(0)=\int_{\mathbb{R}^{n-1}}\left(12 \psi|\bar{\nabla} \psi|^{2} \bar{\Delta} \psi+2 \psi_{t}^{4}-12|\bar{\nabla} \psi|^{2} \psi_{t}^{2}+6|\bar{\nabla} \psi|^{4}\right) d x
$$

Subtracting (5-6) from the sum of (5-3), (5-7), (5-9), we conclude that

$$
\begin{aligned}
I^{\prime \prime \prime \prime}(0) & =-3 \alpha_{n} \frac{(n-2)^{3} n\left(3 n^{3}+2 n^{2}-13 n-6\right)}{2(n+1)(2 n+3)(2 n+1)} E \\
& =-\frac{3 \pi^{n / 2} \Gamma\left(\frac{3 n+1}{2}\right)(n-2)^{4} n^{2}\left(3 n^{3}+2 n^{2}-13 n-6\right)}{\Gamma\left(n+\frac{1}{2}\right) \Gamma(n) 2^{2 n+1}(n+1)(2 n+3)(2 n+1)}<0 .
\end{aligned}
$$

The proof of Theorem 4 is now complete.

Remark. Equation (3-20) can be written as

$$
\begin{aligned}
I^{\prime \prime \prime \prime}(0)=\int_{\mathbb{R}^{n-1}}\left(12 \psi|\bar{\nabla} \psi|^{2} \bar{\Delta} \psi+24 \psi \psi_{t}\left\langle\bar{\nabla} \psi, \bar{\nabla} \psi_{t}\right\rangle\right. \\
+12\left(|\bar{\nabla} \psi|^{2}-\psi_{t}^{2}\right) \mathscr{P}\left(\psi \psi_{t}\right)_{t}+2 \psi_{t}^{4}+12|\bar{\nabla} \psi|^{2} \psi_{t}^{2} \\
\left.+6|\bar{\nabla} \psi|^{4}-12 \theta_{t}\left(|\bar{\nabla} \psi|^{2}-\psi_{t}^{2}\right)-24\langle\bar{\nabla} \psi, \bar{\nabla} \theta\rangle \psi_{t}\right) d x
\end{aligned}
$$


Note that $\theta$ occurs linearly in this integral and only in the last two terms. Thus since we can always multiply $\theta$ by a large positive or negative constant it would suffice to prove the following conjecture in order to obtain examples where $I^{\prime \prime \prime \prime}(0)$ has both signs.

Conjecture 1. Given $0 \not \equiv \psi \in C_{0}^{\infty}\left(\mathbb{R}^{n-1}\right), n \geq 3$, with $I^{\prime \prime \prime}(0)=0$, there exists $\theta \in C_{0}^{\infty}\left(\mathbb{R}^{n-1}\right)$ with

$$
\begin{aligned}
0 & \neq \int_{\mathbb{R}^{n-1}}\left(12 \theta_{t}\left(|\bar{\nabla} \psi|^{2}-\psi_{t}^{2}\right)+24\langle\bar{\nabla} \psi, \bar{\nabla} \theta\rangle \psi_{t}\right) d x \\
& =\int_{\mathbb{R}^{n-1}}\left(12 \theta_{t}\left(|\bar{\nabla} \psi|^{2}-\psi_{t}^{2}\right)-12 \theta\left(|\bar{\nabla} \psi|^{2}-\psi_{t}^{2}\right)_{t}\right) d x \\
& =\int_{D(0)} 12 \theta \bar{\Delta}\left(|\bar{\nabla} \psi|^{2}-\psi_{t}^{2}\right) d x .
\end{aligned}
$$

Let

$$
\theta(x, t)=\frac{\Gamma\left(\frac{n}{2}\right)}{\pi^{n / 2}(2-n)}\left(|x-y|^{2}+t^{2}\right)^{1-n / 2}
$$

for fixed $y \in \mathbb{R}^{n-1}$. Using this $\theta$ in Conjecture 1 and letting $t \rightarrow 0$ through positive values we see from well-known facts about the Poisson integral that the conjecture is equivalent to the inequality

$$
\left(|\bar{\nabla} \psi|^{2}-\psi_{t}^{2}\right)(y, 0) \not \equiv 2 \sum_{i=1}^{n-1} R_{i}\left(\psi_{x_{i}} \psi_{t}\right)(y, 0) \quad \text { when } y \in \mathbb{R}^{n-1},
$$

where each $R_{i}(x)=\left(\Gamma\left(\frac{n}{2}\right) / \pi^{n / 2}\right)\left(x_{i} /|x|\right)$, for $1 \leq i \leq n-1$, is a Riesz transform and $R_{i} f$ denotes the convolution of $R_{i}$ with $f$. A less likely conjecture is:

Conjecture 2. Given $\psi \in C_{0}^{\infty}\left(\mathbb{R}^{n-1}\right)$ with $I^{\prime \prime \prime}(0)=0$ and $\theta \equiv 0$, it is always true that the integral in (3-20) is positive.

The following examples, all with $n=4$, lend some credence to these conjectures. The first example involves

$$
\psi=\frac{1}{r^{2}+(1+t)^{2}}-\frac{a^{2}}{r^{2}+(t+a)^{2}},
$$

where $0<a<1$ and $r^{2}=x^{2}+y^{2}+z^{2}$. For this $\psi$ we find, using residues, that $I^{\prime \prime \prime}(0)=0$ for all $a$. The remaining calculations in this section are all from Maple. The fourth derivatives are

$$
\begin{aligned}
I_{\theta=0}^{\prime \prime \prime \prime}(0) & =\frac{1}{8} \frac{\pi^{2}\left(3 a^{2}+22 a+3\right)(a-1)^{4}}{a(a+1)^{5}}, \\
I_{\theta=\psi \psi_{t}}^{\prime \prime \prime \prime}(0) & =\frac{1}{4} \frac{\left(3 a^{2}+26 a+3\right)(a-1)^{4} \pi^{2}}{a(a+1)^{5}} .
\end{aligned}
$$


These derivatives are positive and unequal, supporting both conjectures. To find $I_{\theta=0}^{\prime \prime \prime \prime}(0)$ for this example we needed to calculate $g_{\varepsilon \varepsilon}$, which we did by using partial fractions on $2 \psi \psi_{t}$ and then solving a system of equations based on the observation that $g_{\varepsilon \varepsilon}$ can be realized as a linear combination of $t$ derivatives of

$$
u=\frac{1}{r^{2}+(t+1)^{2}} \quad \text { and } \quad v=\frac{1}{r^{2}+(t+a)^{2}} .
$$

One obtains more evidence for Conjectures 1 and 2 from the following examples, which also imply, along with the preceding one, that $I_{\theta=\psi \psi_{t}}^{\prime \prime \prime \prime}(0)$ may have either sign. All the examples involve linear combinations of derivatives of the $u$ just defined. Again, this makes finding $g_{\varepsilon \varepsilon}$ possible when $\theta=0$ using derivatives of $u$. We always use an odd number of $x$ derivatives so that $I^{\prime \prime \prime}(0)=0$.

Here is a table of some derivatives.

$$
\begin{array}{ccc}
\psi & I_{\theta=0}^{\prime \prime \prime \prime}(0) / \pi^{2} & I_{\theta=\psi \psi_{t}}^{\prime \prime \prime \prime}(0) / \pi^{2} \\
u_{x}=-2 x u^{2} & \frac{141}{320} & -\frac{83}{40} \\
u_{x t}=8 x(t+1) u^{3} & \frac{103899}{1600} & -\frac{48051}{160} \\
u_{x x x}=-48 x^{3} u^{4}+24 x u^{3} & \frac{302804163}{125440} & -\frac{6567399}{640} \\
u_{t t x}=-48 u^{4}(t+1)^{2} x+8 u^{3} x & \frac{462648861}{17920} & -\frac{75385143}{640} \\
u_{x x x x x}=-3840 u^{6} x^{5}+3840 u^{5} x^{3}-720 u^{4} x & \frac{4739410625625}{5632} & -\frac{423800960625}{128}
\end{array}
$$

We also computed many $I_{\theta=0}^{\prime \prime \prime \prime}(0)$ and $I_{\theta=\psi \psi_{t}}^{\prime \prime \prime \prime}(0)$ for many linear combinations $\psi$. For example, for $\psi=u_{x t}+a u_{x x x}$, we get

$$
I_{\theta=0}^{\prime \prime \prime \prime}(0)=\frac{3 \pi^{2}}{627200}\left(504673605 a^{4}+760731228 a^{3}+444523278 a^{2}+120281560 a+13576136\right)
$$

and

$$
I_{\theta=\psi \psi}^{\prime \prime \prime \prime} \psi_{t}(0)=\frac{-3 \pi^{2}}{4480}\left(15323931 a^{4}+23718213 a^{3}+14150139 a^{2}+3890212 a+448476\right) .
$$

In all these examples of linear combinations we found that $I_{\theta=0}^{\prime \prime \prime \prime}(0)>0$, supporting Conjecture 2, while $I_{\theta=\psi \psi_{t}}^{\prime \prime \prime \prime}(0)<0$, supporting Conjecture 1, and showing along with the example at the bottom of page 163 that $I_{\theta=\psi \psi_{t}}^{\prime \prime \prime \prime}(0)$ may have either sign.

Another way to generate examples is to use separation of variables in $(r, t)$. Then for any $\lambda>0$ the functions $e^{-\lambda t} \sin (\lambda r) / r$ are harmonic in $\mathbb{R}_{+}^{4}$ and, with appropriate integrability,

$$
g(r, t)=\sqrt{2 / \pi} \int_{0}^{\infty} \frac{e^{-\lambda t} \sin (\lambda r)}{r} f(\lambda) d \lambda
$$

represents the harmonic function with boundary values

$$
g(r, 0)=\sqrt{2 / \pi} \int_{0}^{\infty} \frac{\sin (\lambda r)}{r} f(\lambda) d \lambda
$$


So one can calculate examples in the variables $(r, t)$ using Fourier sine transforms when $n=4$, or by using Hankel transforms in higher dimensions. The examples we worked out did not lead to anything new.

Finally let $\mu, \tilde{\mu}$ denote (as in Section 1) harmonic measure defined with respect to the above $D, \mathbb{R}^{n} \backslash \bar{D}$. Since both these measures are doubling measures it follows easily from a monotonicity formula of [Alt et al. 1984] (see also [Friedland and Hayman 1976]) that if $x \in \partial D, 0 \leq r \leq 1$, then

$$
\mu(B(x, r)) \tilde{\mu}(B(x, r)) \leq c r^{2 n-2},
$$

where $c$ depends only on $n$. Now for $\mu, D$ as in Corollary 1 and almost every $x \in \operatorname{supp} \mu$ it follows from Wolff's argument and Theorem 3 that

$$
\lim _{r \rightarrow 0} \frac{\ln \mu(B(x, r))}{\ln r}<n-1 .
$$

A similar statement holds for $\tilde{\mu}$. Using these equalities in the monotonicity formula we reach a contradiction unless $\mu$ and $\tilde{\mu}$ are mutually singular. On the other hand there is no contradiction to the above inequality if $\mu, \tilde{\mu}, D$ are as in Corollary 2. Thus we conjecture:

Conjecture 3. There exists a Wolff snowflake for which $\mu, \tilde{\mu}$ are not mutually singular.

In two dimensions (see [Bishop et al. 1989]) a necessary and sufficient condition for these measures to be mutually singular in a Jordan domain is that the set of boundary points with tangents has $H^{1}$ measure zero. Clearly Wolff snowflakes have this property, so $\mu, \tilde{\mu}$ are always mutually singular in $\mathbb{R}^{2}$.

\section{References}

[Ahlfors 1978] L. V. Ahlfors, Complex analysis: An introduction to the theory of analytic functions of one complex variable, 3rd ed., McGraw-Hill, New York, 1978. MR 80c:30001

[Alt et al. 1984] H. W. Alt, L. A. Caffarelli, and A. Friedman, "Variational problems with two phases and their free boundaries", Trans. Amer. Math. Soc. 282:2 (1984), 431-461. MR 85h:49014 Zbl 0844.35137

[Batakis 1996] A. Batakis, "Harmonic measure of some Cantor type sets", Ann. Acad. Sci. Fenn. Math. 21:2 (1996), 255-270. MR 97f:31002 Zbl 0849.31005

[Bishop et al. 1989] C. J. Bishop, L. Carleson, J. B. Garnett, and P. W. Jones, "Harmonic measures supported on curves”, Pacific J. Math. 138:2 (1989), 233-236. MR 90d:30069

[Bourgain 1987] J. Bourgain, "On the Hausdorff dimension of harmonic measure in higher dimension”, Invent. Math. 87:3 (1987), 477-483. MR 88b:31004 Zbl 0616.31004

[Carleson 1985] L. Carleson, "On the support of harmonic measure for sets of Cantor type", Ann. Acad. Sci. Fenn. Ser. A I Math. 10 (1985), 113-123. MR 87b:31002 Zbl 0593.31004

[Friedland and Hayman 1976] S. Friedland and W. K. Hayman, "Eigenvalue inequalities for the Dirichlet problem on spheres and the growth of subharmonic functions", Comment. Math. Helv. 51:2 (1976), 133-161. MR 54 \#568 Zbl 0339.31003 
[Helms 1969] L. L. Helms, Introduction to potential theory, Pure and Applied Mathematics 22, Wiley, New York, 1969. MR 41 \#5638

[Jones and Wolff 1988] P. W. Jones and T. H. Wolff, "Hausdorff dimension of harmonic measures in the plane", Acta Math. 161:1-2 (1988), 131-144. MR 90j:31001 Zbl 0667.30020

[Kaufman and Wu 1985] R. Kaufman and J.-M. Wu, "On the snowflake domain”, Ark. Mat. 23:1 (1985), 177-183. MR 86m:31002

[Lewis and Vogel 1991] J. L. Lewis and A. Vogel, "On pseudospheres", Rev. Mat. Iberoamericana 7:1 (1991), 25-54. MR 92g:31006 Zbl 0734.31006

[Lewis and Vogel 2001] J. L. Lewis and A. Vogel, "On pseudospheres that are quasispheres", Rev. Mat. Iberoamericana 17:2 (2001), 221-255. MR 2003b:30029 Zbl 1005.30020

[Makarov 1985] N. G. Makarov, "On the distortion of boundary sets under conformal mappings", Proc. London Math. Soc. (3) 51:2 (1985), 369-384. MR 87d:30012 Zbl 0573.30029

[Stein 1970] E. M. Stein, Singular integrals and differentiability properties of functions, Princeton Mathematical Series 30, Princeton University Press, Princeton, 1970. MR 44 \#7280

[Volberg 1993] A. Volberg, "On the dimension of harmonic measure of Cantor repellers", Michigan Math. J. 40:2 (1993), 239-258. MR 95d:30043 Zbl 0797.30022

[Wolff 1995] T. H. Wolff, "Counterexamples with harmonic gradients in $\mathbf{R}^{3}$ ", pp. 321-384 in Essays on Fourier analysis in honor of Elias M. Stein (Princeton, 1991), Princeton Math. Ser. 42, Princeton Univ. Press, Princeton, NJ, 1995. MR 95m:31010

Received May 28, 2002.

JOHN L. LEWIS

DePartMENT OF MATHEMATICS

UNIVERSITY OF KENTUCKY

LEXINGTON, KY 40506-0027

john@ms.uky.edu

GREGORY C. VERCHOTA

DEPARTMENT OF MATHEMATICS

SYRACUSE UNIVERSITY

SYRACUSE, NY 13244

gverchot@syr.edu

ANDREW L. VogeL

DEPARTMENT OF MATHEMATICS

SYRACUSE UNIVERSITY

SYRACUSE, NY 13244

alvogel@syr.edu 